\title{
Planck pre-launch status: Calibration of the Low Frequency Instrument flight model radiometers
}

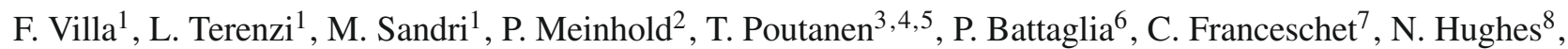
M. Laaninen ${ }^{8}$, P. Lapolla ${ }^{6}$, M. Bersanelli ${ }^{7}$, R. C. Butler ${ }^{1}$, F. Cuttaia ${ }^{1}$, O. D’Arcangelo ${ }^{9}$, M. Frailis ${ }^{10}$, E. Franceschi ${ }^{1}$, S. Galeotta ${ }^{10}$, A. Gregorio ${ }^{11}$, R. Leonardi ${ }^{2}$, S. R. Lowe ${ }^{12}$, N. Mandolesi ${ }^{1}$, M. Maris ${ }^{10}$, L. Mendes ${ }^{13}$, A. Mennella ${ }^{7}$, G. Morgante ${ }^{1}$, L. Stringhetti ${ }^{1, \star}$, M. Tomasi ${ }^{7}$, L. Valenziano $^{1}$, A. Zacchei ${ }^{10}$, A. Zonca ${ }^{14}$, B. Aja ${ }^{15}$, E. Artal ${ }^{15}$, M. Balasini ${ }^{6}$, T. Bernardino ${ }^{16}$, E. Blackhurst ${ }^{12}$, L. Boschini ${ }^{6}$, B. Cappellini ${ }^{14}$, F. Cavaliere ${ }^{7}$, A. Colin ${ }^{16}$, F. Colombo ${ }^{6}$, R. J. Davis ${ }^{12}$, L. De La Fuente ${ }^{15}$, J. Edgeley ${ }^{12}$, T. Gaier ${ }^{17}$, A. Galtress ${ }^{12}$, R. Hoyland ${ }^{18}$, P. Jukkala ${ }^{8}$, D. Kettle ${ }^{12}$, V.-H. Kilpia ${ }^{8}$, C. R. Lawrence ${ }^{16}$, D. Lawson ${ }^{12}$, J. P. Leahy ${ }^{12}$, P. Leutenegger ${ }^{6}$, S. Levin ${ }^{16}$, D. Maino ${ }^{7}$, M. Malaspina ${ }^{1}$, A. Mediavilla ${ }^{15}$, M. Miccolis ${ }^{6}$, L. Pagan ${ }^{6}$, J. P. Pascual ${ }^{15}$, F. Pasian ${ }^{10}$, M. Pecora ${ }^{6}$, M. Pospieszalski ${ }^{19}$, N. Roddis ${ }^{12}$, M. J. Salmon ${ }^{16}$, M. Seiffert ${ }^{17}$, R. Silvestri ${ }^{6}$, A. Simonetto ${ }^{9}$, P. Sjoman ${ }^{8}$, C. Sozzi ${ }^{9}$, J. Tuovinen ${ }^{20}$, J. Varis ${ }^{20}$, A. Wilkinson ${ }^{12}$, and F. Winder ${ }^{12}$

(Affiliations can be found after the references)

Received 9 July 2009 / Accepted 3 May 2010

ABSTRACT

The Low Frequency Instrument (LFI) on-board the ESA Planck satellite carries eleven radiometer subsystems, called radiometer chain assemblies (RCAs), each composed of a pair of pseudo-correlation receivers. We describe the on-ground calibration campaign performed to qualify the flight model RCAs and to measure their pre-launch performances. Each RCA was calibrated in a dedicated flight-like cryogenic environment with the radiometer front-end cooled to $20 \mathrm{~K}$ and the back-end at $300 \mathrm{~K}$, and with an external input load cooled to $4 \mathrm{~K}$. A matched load simulating a blackbody at different temperatures was placed in front of the sky horn to derive basic radiometer properties such as noise temperature, gain, and noise performance, e.g. $1 / f$ noise. The spectral response of each detector was measured as was their susceptibility to thermal variation. All eleven LFI RCAs were calibrated. Instrumental parameters measured in these tests, such as noise temperature, bandwidth, radiometer isolation, and linearity, provide essential inputs to the Planck-LFI data analysis.
\end{abstract}

Key words. cosmic microwave background - space vehicles: instruments - instrumentation: detectors - techniques: miscellaneous

\section{Introduction}

The Planck mission ${ }^{1}$ has been developed to provide a deep, fullsky image of the cosmic microwave background (CMB) in both temperature and polarization. Planck incorporates an unprecedented combination of sensitivity, angular resolution and spectral range - spanning from centimeter to sub-millimeter wavelengths - by integrating two complementary cryogenic instruments in the focal plane of the Planck telescope. The Low Frequency Instrument (LFI) covers the region below the CMB blackbody peak in three frequency bands centered at 30, 44 and $70 \mathrm{GHz}$. The spectral range of the LFI is also suitable for a wealth of galactic and extragalactic astrophysics. The LFI maps will address studies of diffuse Galactic free-free and synchrotron emission, emission from spinning dust grains, and discrete Galactic radio sources. Extragalactic radio sources will

* Present address: Astrium GmbH, Friedrichshafen, Germany.

1 Planck (http://www.esa.int/Planck) is a project of the European Space Agency - ESA - with instruments provided by two scientific Consortia funded by ESA member states (in particular the lead countries: France and Italy) with contributions from NASA (USA), and telescope reflectors provided in a collaboration between ESA and a scientific Consortium led and funded by Denmark. also be observed, particularly those with flat or strongly inverted spectra, peaking at mm wavelengths. Furthermore, the Planck scanning strategy will also allow monitoring of radio source variability on a variety of time scales. While these are highly interesting astrophysical objectives, the LFI design and calibration is driven by the main Planck scientific scope, i.e., CMB science.

The LFI is an array of cryogenic radiometers based on indium phospide (InP) cryogenic HEMT low noise amplifiers (Bersanelli et al. 2010). The array is composed of 22 pseudocorrelation radiometers mounted in eleven independent radiometer units called "radiometer chain assemblies" (RCAs), two centered at $30 \mathrm{GHz}$, three at $44 \mathrm{GHz}$ and six at $70 \mathrm{GHz}^{2}$. To optimize the sensitivity and minimize the power dissipation in the front end, each RCA is split into a front-end module (FEM), cooled to $20 \mathrm{~K}$, and a back-end module (BEM), operating at $300 \mathrm{~K}$, connected by a set of composite waveguides.

Accurate calibration is mandatory for optimal operation of the instrument during the full-sky survey and for measuring parameters that are essential for the Planck data analysis.

\footnotetext{
2 The chains are numbered as RCAXX where $\mathrm{XX}$ is a number from 18 to 23 for the $70 \mathrm{GHz}$ RCAs, from 24 to 26 for the RCAs at $44 \mathrm{GHz}$, and from 27 to 28 for the RCAs at $30 \mathrm{GHz}$.
} 


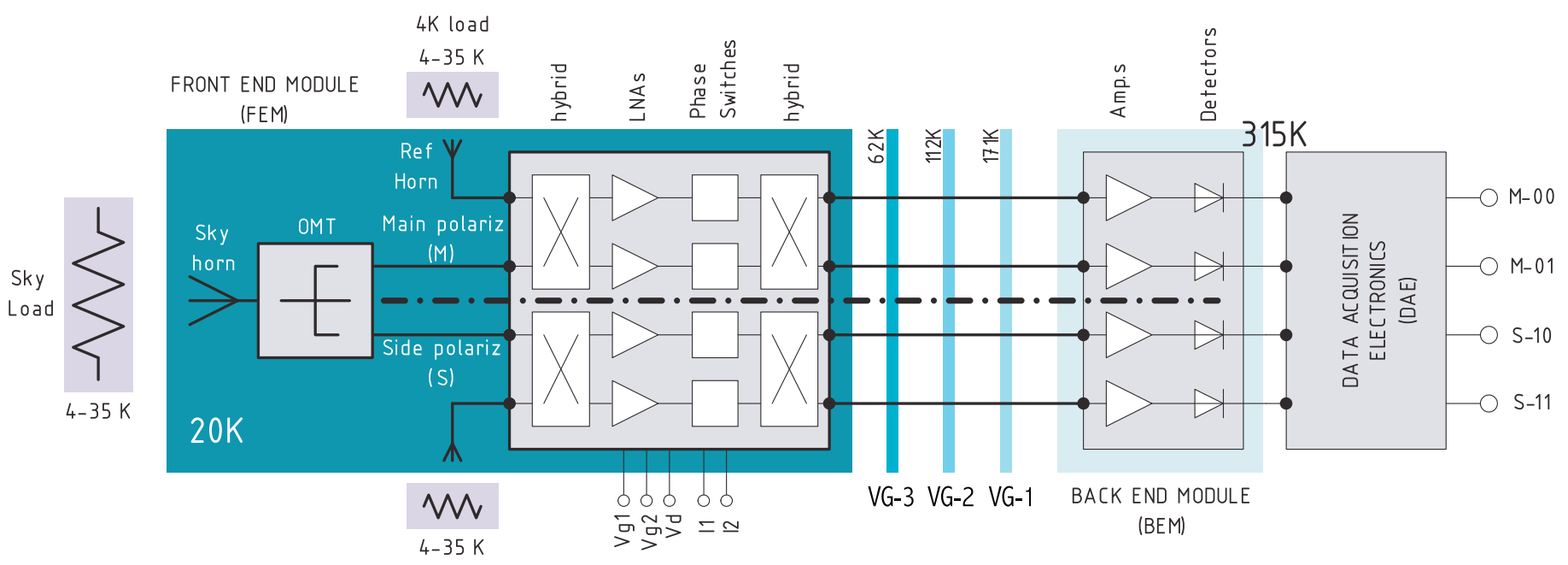

Fig. 1. Scheme of the RCA and its flight-like thermal interfaces. The FEM is at $20 \mathrm{~K}$, while the BEM is at $315 \mathrm{~K}$. At 30 and $44 \mathrm{GHz}$ the thermal interface attached to the third and coldest V-groove (VG-3) is controlled at a temperature near $60 \mathrm{~K}$, while for the $70 \mathrm{GHz}$ RCA the VG-3 interface was not controlled in temperature. Both loads - the sky load and the reference load - are controlled in a temperature of approximately $4 \mathrm{~K}$ to $35 \mathrm{~K}$.

The LFI calibration strategy has been based on a complementary approach that includes both pre-launch and post-launch activities. On-ground measurements were performed at all-unit and sub-unit levels, both for qualification and performance verification. Each single FEM and BEM as well as the passive components (feed horn, orthomode transducers, waveguides, $4 \mathrm{~K}$ reference loads) were tested in a stand-alone configuration before they were integrated into the RCA units.

The final scientific calibration of the LFI was carried out at two different integration levels, depending on the measured parameter. First each RCA was tested independently in dedicated cryofacilities, which are capable of reaching a temperature of $\sim 4 \mathrm{~K}$ at the external input loads. These conditions were necessary for an accurate measurement of key parameters such as system noise temperature, bandwidth, radiometer isolation, and linearity. Subsequently, the eleven RCAs were integrated into the full LFI instrument (the so-called radiometer array assembly, RAA) and tested as a complete instrument system in a large cryofacility, with highly stable input loads cooled down to $20 \mathrm{~K}$.

We reports on the calibration campaign of the RCAs, while Mennella et al. (2010) report on the RAA calibration. The parameters derived here are crucial for the Planck-LFI scientific analysis. Noise temperatures, bandwidths, and radiometer isolation provide essential information to construct an adequate noise model, which is needed as an input to the map-making process. Any non-linearity of the instrument response must be accurately measured, because corrections may be needed in the data analysis, particularly for observations of strong sources such as planets (crucial for in-flight beam reconstruction) and the Galactic plane. As part of the RCA testing, we also performed an endto-end measurement of the bandshape inside the cryofacility. Finally, for comparison and as a consistency check, the RCA test plan also included measurement of parameters whose primary calibration relies on the RAA test campaign, such as optimal radiometer bias (tuning), $1 / f$ noise (knee frequency and slope), gain, and thermal susceptibility.

The 30 and $44 \mathrm{GHz}$ RCAs were integrated and tested in Thales Alenia Space Italia (TAS-I), formerly Laben, from the beginning of January 2006 to end of May 2006 using a dedicated cryofacility (Terenzi et al. 2009b) to reproduce flight-like thermal interfaces and input loads. The $70 \mathrm{GHz}$ RCAs were integrated and calibrated in Yilinen Electronics (Finland) from the end of April 2005 to mid February 2006 with a similar cryofacility, but with simplified thermal interfaces (Terenzi et al. 2009b). The differences between the two cryofacilities resulted in slightly different test procedures, because the temperatures were not controlled in the same way.

Section 2 of the paper describes the concept of RCA calibration, while Sect. 3 illustrates the two cryofacilities. In Sect. 4 we describe each test, the methods used in the analysis and the results. The conclusions are given in Sect. 5 .

\section{Main concepts and calibration logic}

\subsection{Radiometer chain assembly description}

A diagram of an RCA is shown in Fig. 1. A corrugated feed horn (Villa et al. 2009), which collects the radiation from the telescope, $T_{\text {sky }}$, is connected to an ortho-mode transducer (D'Arcangelo et al. 2009b), which divides the signal into two orthogonal polarizations, namely "M" (main) and "S" (side) branches. The OMT is connected to the front-end section (Davis et al. 2009; Varis et al. 2009), in which each polarization of the sky signal is mixed with the signal from a stable reference load, $T_{\text {ref }}$, via a hybrid coupler (Valenziano et al. 2009). The signal is then amplified by a factor $G_{\text {fe }}$ and shifted in phase by $0-180$ degrees at $8 \mathrm{kHz}$ synchronously with the acquisition electronics. Finally, a second hybrid coupler separates the input sky signal from the reference load signal.

$1.75 \mathrm{~m}$ long waveguides (D’Arcangelo et al. 2009a) are connected to the FEM in bundles of four elements providing the thermal break between $20 \mathrm{~K}$ and $300 \mathrm{~K}$ where the ambient temperature back-end section of the radiometer is located (Artal et al. 2009; Varis et al. 2009). The waveguides are thermally attached to the three thermal shields of the satellite, the V-grooves. They act as radiators to passively cool down the payload to about $50 \mathrm{~K}$. They drive the thermal gradient along the waveguides.

Inside the BEM the signal is further amplified by $G_{\text {be }}$ and then detected by four output detector diodes ${ }^{3}$. In nominal conditions, each of the four diodes detects a voltage alternatively (each $122 \mu \mathrm{s}$ which corresponds to $1 / 8192 \mathrm{~Hz}^{-1}$ ) proportional

\footnotetext{
3 According to the name convention, diodes refer to the Mainpolarization are labeled as $M-\otimes \theta, M-01$ and those referring to the Sidepolarization are labeled as $\mathrm{S}-10, \mathrm{~S}-11$.
} 
by a factor $a$ to the sky load and reference load temperature. By differencing these two signals a very stable output is obtained, which allows the measurement of very faint signals.

Assuming negligible mismatches between the two radiometer legs and within the phase switch, the differenced radiometer output at each detector averaged over the bandwidth $\beta$ can be written in terms of overall gain, $G_{\text {tot }}$ in units of $\mathrm{V} / \mathrm{K}$, and noise temperature, $T_{\mathrm{N}}$, as

$$
\begin{aligned}
V_{\text {out }}= & G_{\text {tot }}\left[\left(\widetilde{T}_{\text {sky }}+T_{\mathrm{N}}\right)-r \cdot\left(\widetilde{T}_{\text {ref }}+T_{\mathrm{N}}\right)\right] \\
& \times G_{\text {tot }}=a \cdot k \cdot \beta \cdot G_{\mathrm{fe}} L_{\mathrm{wg}} G_{\mathrm{be}} \\
& \times T_{\mathrm{N}} \simeq T_{\mathrm{N}}^{(\mathrm{fe})}+\frac{T_{\mathrm{N}}^{(\mathrm{be})}}{G_{\mathrm{fe}}} .
\end{aligned}
$$

Here we further define the waveguide losses as $L_{\mathrm{wg}}$, the front-end noise temperature a $T_{\mathrm{N}}^{(\mathrm{fe})}$, and the back-end noise temperature as $T_{\mathrm{N}}^{(\mathrm{be})}$, and with $k$, the Boltzmann constant. The noise contribution of the waveguides due to its attenuation is negligible and not considered here.

The ohmic losses of the feedhorn - OMT assembly, $L_{\mathrm{fo}}$, and of the $4 \mathrm{~K}$ Reference load system, $L_{4} \mathrm{~K}$, modify the actual sky and reference load through the following equations

$$
\begin{aligned}
& \widetilde{T}_{\text {sky }}=\frac{T_{\text {sky }}}{L_{\text {fo }}}+\left(1-\frac{1}{L_{\text {fo }}}\right) T_{\text {phys }} \\
& \widetilde{T}_{\text {ref }}=\frac{T_{\text {ref }}}{L_{4 \mathrm{~K}}}+\left(1-\frac{1}{L_{4} \mathrm{~K}}\right) T_{\text {phys }},
\end{aligned}
$$

where $T_{\text {phys }}$ its the physical temperature (close to $20 \mathrm{~K}$ at operational conditions).

The $r$ factor in Eq. (1) is the gain modulation factor calculated as

$r=\frac{\widetilde{T}_{\text {sky }}+T_{\mathrm{N}}}{\widetilde{T}_{\text {ref }}+T_{\mathrm{N}}}$,

which nulls the radiometer output. A more general form of the averaged power output, which takes into account various nonideal behaviors of the radiometer components, can be found in Seiffert et al. (2002) and Mennella et al. (2003). The key parameters needed to reconstruct the required signal (differences of $T_{\text {sky }}$ from one point of the sky to another) are therefore the photometric calibration, $G_{\text {tot }}$, and the gain modulation factor, $r$, which is used to suppress the effect of $1 / f$ noise. Deviations from this first approximation are treated as systematic effects.

\subsection{Signal model}

To better understand the purpose of the calibration, it is useful to write Eqs. (1) to (4) appropriately so that the attenuation coefficients are taken into account in the RCA parameters instead of considering their effects as a target effective temperature. For the sky signal the output can be written as

$V_{\text {out }}^{\text {sky }}=\widetilde{G}_{\text {tot }}^{\text {sky }}\left(T_{\text {sky }}+\widetilde{T}_{\mathrm{N}}^{\text {sky }}\right)$,

$\widetilde{G}_{\mathrm{tot}}^{\mathrm{sky}}=a \cdot k \cdot \beta \frac{1}{L_{\mathrm{fo}}} G_{\mathrm{fe}} \frac{1}{L_{\mathrm{wg}}} G_{\mathrm{be}}$,

$\widetilde{T}_{\mathrm{N}}^{\mathrm{sky}}=T_{\mathrm{N}}+\left[\left(L_{\mathrm{fo}}-1\right) T_{\mathrm{phys}}\right]$,

and equivalently for the reference signal

$V_{\text {out }}^{\text {ref }}=\widetilde{G}_{\text {tot }}^{\text {ref }}\left(T_{\text {ref }}+\widetilde{T}_{\mathrm{N}}^{\text {ref }}\right)$,
$\widetilde{G}_{\mathrm{tot}}^{\mathrm{ref}}=a \cdot k \cdot \beta \frac{1}{L_{4 \mathrm{~K}}} G_{\mathrm{fe}} \frac{1}{L_{\mathrm{wg}}} G_{\mathrm{be}}$,

$\widetilde{T}_{\mathrm{N}}^{\mathrm{ref}}=T_{\mathrm{N}}+\left[\left(L_{4 \mathrm{~K}}-1\right) T_{\mathrm{phys}}\right]$.

Differencing Eqs. (5) and (8) we obtain the differenced (sky ref) output similar to Eq. (1)

$$
\begin{aligned}
V_{\mathrm{out}} & =G_{\mathrm{tot}}^{*}\left[\left(T_{\mathrm{sky}}+\widetilde{T}_{\mathrm{N}}^{\mathrm{sky}}\right)-r^{*}\left(T_{\mathrm{ref}}+\widetilde{T}_{\mathrm{N}}^{\mathrm{ref}}\right)\right], \\
G_{\mathrm{tot}}^{*} & =G_{\mathrm{tot}} \frac{1}{L_{\mathrm{fo}}} \\
r^{*} & =r \frac{L_{\mathrm{fo}}}{L_{4 \mathrm{~K}}}
\end{aligned}
$$

Equations (5), (8), and (11) are the basis for the RCA calibration, because all parameters involved were measured during the RCA test campaign by stimulating each RCA at cryo temperature (close to the operational in-flight conditions) with several known $T_{\text {sky }}$ and $T_{\text {ref values. }}$

\subsection{Radiometer chain assembly calibration plan}

Each RCA calibration included (i) functional tests, to verify the functionality of the RCA; (ii) bias tuning, to set the best amplifier gains and phase switch bias currents for maximum performance (i.e. minimum noise temperature and best radiometer balancing); (iii) basic radiometer property measurements to estimate $G^{*}, \widetilde{T}_{\mathrm{N}}^{\text {sky }}, \widetilde{T}_{\mathrm{N}}^{\text {ref }}$; (iv) noise performance measurements to evaluate $1 / f$, white noise level and the $r^{*}$ parameter; (v) spectral response measurements to derive the relative bandshape; (vi) susceptibility measurements of radiometer thermal variations to estimate the dependence of noise and gain with temperature. The list of tests is given in Table 1 together with a brief description of the purpose of each test.

Although the calibration plan was the same for all RCAs, the differences in the setup between $30 / 44 \mathrm{GHz}$ and $70 \mathrm{GHz}$ resulted in a different test sequence and procedures, which retained the objective of RCA calibration unchanged.

At $70 \mathrm{GHz}$ the RCA tests were carried out in a dedicated cryogenic chamber developed by DA-Design ${ }^{4}$ (formerly Ylinen Electronics), which was capable of accommodating two RCAs at one time. Thus the RCA test campaign was planned for three RCA pairs, namely RCA18 and RCA23, RCA19 and RCA20, RCA2 1 and RCA22. At 30 and $44 \mathrm{GHz}$, only one RCA at a time was calibrated. Due to the different length of the waveguides, the thermal interfaces were not exactly the same for different RCAs, which resulted in a slightly different thermal behavior of the cryogenic chamber and thus slightly different calibration conditions.

\section{Radiometer chain assembly calibration facilities}

In both cases, the calibration facilities used the cryogenic chamber described in Terenzi et al. (2009b), a calibration load, the skyload (Terenzi et al. 2009a), electronic ground support equipment (EGSE) and software (Malaspina et al. 2009). The heart of the EGSE was a breadboard of the LFI flight data acquisition electronics (DAE) with Labwindow ${ }^{\mathrm{TM} 5}$ software to control the power supplies to the FEMs and BEMs, read all the housekeeping parameters and digitize the scientific signal at $8 \mathrm{KHz}$ without any average or time integration. The EGSE sent data continuously to a workstation operating the Rachel (RAdiometer

\footnotetext{
4 http://www.da-design. fi/space

5 http://www .ni.com/lwcvi/
} 
Table 1. Calibration test list.

\begin{tabular}{|c|c|}
\hline TEST ID & DESCRIPTION \\
\hline & FUNCTIONALITY \\
\hline RCA_AMB & Functional test at ambient \\
\hline RCA_CRY & $\begin{array}{c}\text { Functional test at cryo } \\
\text { TUNING }\end{array}$ \\
\hline RCA_TUN & $\begin{array}{l}\text { Gain and offset tuning of the DAE } \\
\text { Tuning of the front end module } \\
\text { (phase switches and gate voltages) } \\
\text { BASIC PROPERTIES }\end{array}$ \\
\hline RCA_OFT & $\begin{array}{l}\text { Radiometer offset } \\
\text { measurement }\end{array}$ \\
\hline RCA_TNG & $\begin{array}{l}\text { Noise temperature and } \\
\text { photometric gain }\end{array}$ \\
\hline RCA_LIS & $\begin{array}{r}\text { Radiometer linearity } \\
\text { NOISE PROPERTIES }\end{array}$ \\
\hline RCA_STn & $\begin{array}{l}\text { Noise performances tests: } \\
\mathrm{WN}, f_{\mathrm{k}} \text { and } \alpha, \beta, r\end{array}$ \\
\hline RCA_UNC & $\begin{array}{l}\text { Verification of the effect } \\
\text { of the radiometer switching } \\
\text { on the noise spectrum }\end{array}$ \\
\hline & BAND PASS RESPONSE \\
\hline RCA_SPR & $\begin{array}{l}\text { Bandpass } \\
\text { SUSCEPTIBILITY }\end{array}$ \\
\hline RCA_THF & $\begin{array}{l}\text { Susceptibility to } \\
\text { FEM temperature variations }\end{array}$ \\
\hline RCA_THB & $\begin{array}{l}\text { Susceptibility to } \\
\text { BEM Temperature variations }\end{array}$ \\
\hline RCA_THV & $\begin{array}{l}\text { Susceptibility to V-groove } \\
\text { temperature variations }\end{array}$ \\
\hline
\end{tabular}

Notes. The first column reports the test identification. In the second column the purpose of each test is described; WN is the white noise level; $f_{\mathrm{k}}, \alpha$ are the $1 / f$ knee frequency and slope respectively; $\beta$ is the equivalent radiometer bandwidth derived from noise; $r$ is the modulation factor. Apart from the first test RCA_AMB, which is performed at ambient temperature, all the other tests are performed at the operational temperature (i.e. at a temperature as close as possible to in-orbit conditions).

CHain EvaLuator) software for quick-look analysis and data storage (Malaspina et al. 2009). The data files were stored in FITS format. As two chains were calibrated at the same time at $70 \mathrm{GHz}$, separate EGSEs and analysis workstations were used for each RCA. Below the cryofacilities and skyloads are summarized with the emphasis on the issues related to the analysis of the calibration data.

\subsection{The cryofacility for the 30 and $44 \mathrm{GHz} R C A s$}

The chamber with its overall dimensions of $2.0 \times 1.2 \times 1.0 \mathrm{~m}^{3}$ was able to accept one RCA at a time. The chamber was designed to allow the pressure to reach less than $10^{-5} \mathrm{mBar}$, and contained seven thermal interfaces to reproduce the flight-like thermal conditions of an RCA. During tests it was possible to control and stabilize the BEM temperature, the waveguide-to-spacecraft interface temperature, and the FEM temperature. In addition the two reference targets (the reference load and the sky load) were controlled in temperatures in the range $4-35 \mathrm{~K}$ to allow temperature stepping for radiometer linearity tests (RCA_LIS). In addition to the electrical connections for the DAE breadboard and to control the thermal interfaces, two thermal-vacuum feedthroughs (one for the $30 \mathrm{GHz}$ and the other one for the $44 \mathrm{GHz}$ RCAs) with Kapton windows were provided to allow access for the RF signal for the bandpass tests (RCA_SPR).
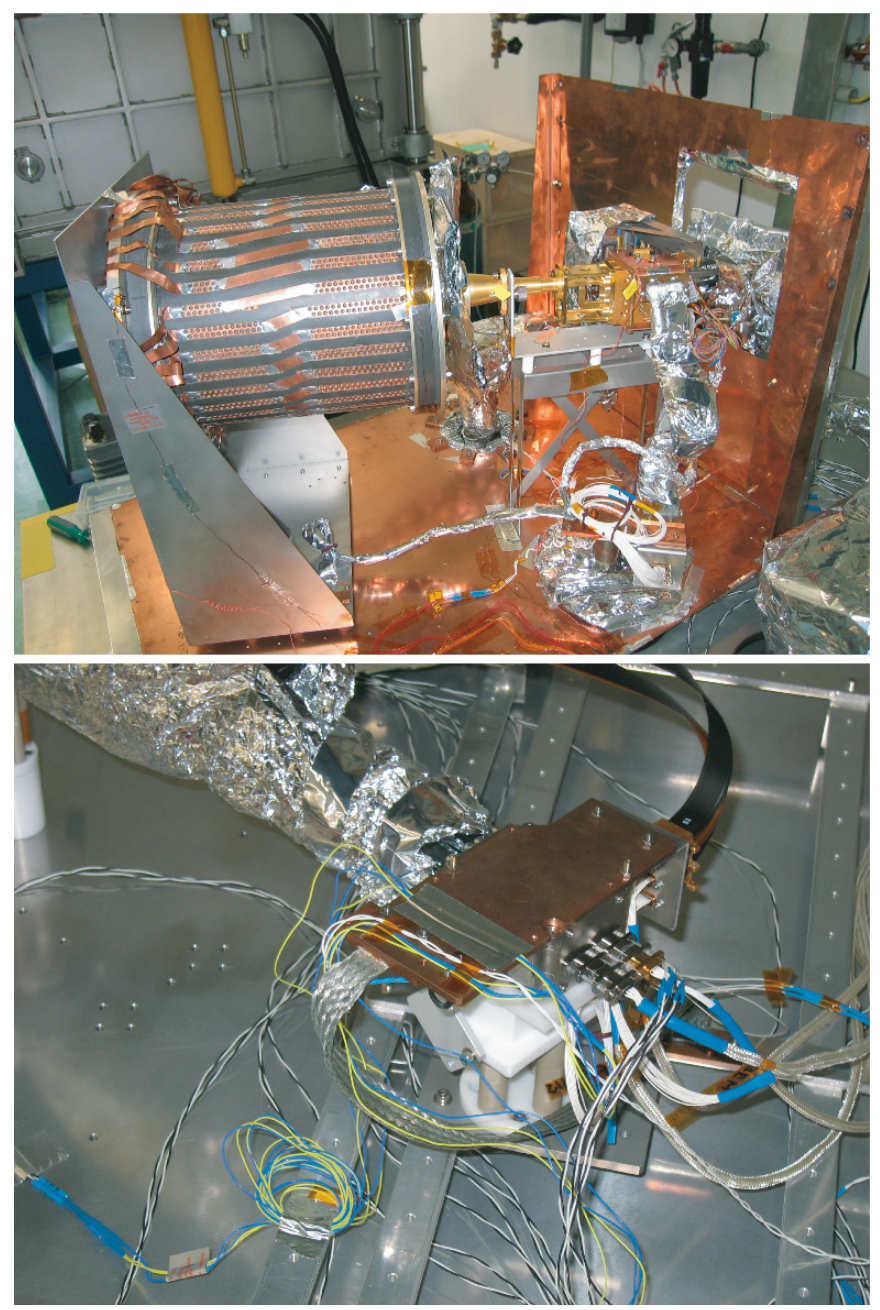

Fig. 2. Radiometer chain assembly integrated into the 30 and $44 \mathrm{GHz}$ cryofacility for calibration. In the picture at the top the skyload facing the horn is visible together with the FEM insulated from the $50 \mathrm{~K}$ shroud (the copper box). In the bottom picture the BEM and its thermal interface are shown. See the text for details of the cryochamber.

During the RCA27 and RCA28 calibrations an uncertainty in the reference targets' temperature was experienced. A visual inspection of the cryochamber after the RCA28 test gave a possible explanation and in the RCA27 test, an additional sensor was put on the back of one of the reference targets in order to verify the probable source of the problem. The observed behavior was consistent with an excess heat flow through the $4 \mathrm{~K}$ reference load (4KRL), via its insulated support caused by a contact created during cooldown. A dedicated thermal model was thus developed to derive the Eccosorb 4KRL temperature, $T_{\text {ref }}$ from the back plate controller sensor temperature, $T_{\mathrm{ref}}^{\mathrm{ctrl}}$ (Terenzi et al. $2009 b)$. A quadratic fit was found with $T_{\text {ref }}=a+b \cdot T_{\mathrm{ref}}^{\mathrm{ctrl}}+c$. $\left(T_{\text {ref }}^{\mathrm{ctrl}}\right)^{2}$ for each pair of detectors coupled to the same radiometer arm and for each $30 \mathrm{GHz}$ RCA. The coefficients derived from the fit are shown in Table 2.

\subsection{Sky load at 30 and $44 \mathrm{GHz}$}

The calibrator consisted of a cylindrical cavity with walls covered in Eccosorb CR $110^{6}$ (see Fig. 6). The back face of the

${ }_{6}^{6}$ Emerson \& Cuming, http : www . eccosorb. com 
Table 2. Reference load target temperature.

\begin{tabular}{ccc}
\hline \hline & \multicolumn{2}{c}{ RCA27 } \\
& $\mathrm{M}$ & $\mathrm{S}$ \\
\hline$a$ & $5.7 \pm 0.2$ & $2.5 \pm 0.1$ \\
$b$ & $0.58 \pm 0.02$ & $0.81 \pm 0.01$ \\
$c$ & $0.00686 \pm 5.8 \times 10^{-4}$ & $0.00322 \pm 3.2 \times 10^{-4}$ \\
\hline \multicolumn{3}{c}{ RCA28 } \\
\multicolumn{3}{c}{$\mathrm{S}$} \\
\hline$a$ & $2.47 \pm 0.05$ & $5.50 \pm 0.09$ \\
$b$ & $0.799 \pm 0.007$ & $0.57 \pm 0.01$ \\
$c$ & $0.00407 \pm 2.5 \times 10^{-4}$ & $0.00831 \pm 4.1 \times 10^{-4}$ \\
\hline
\end{tabular}

Notes. Quadratic fit coefficients.

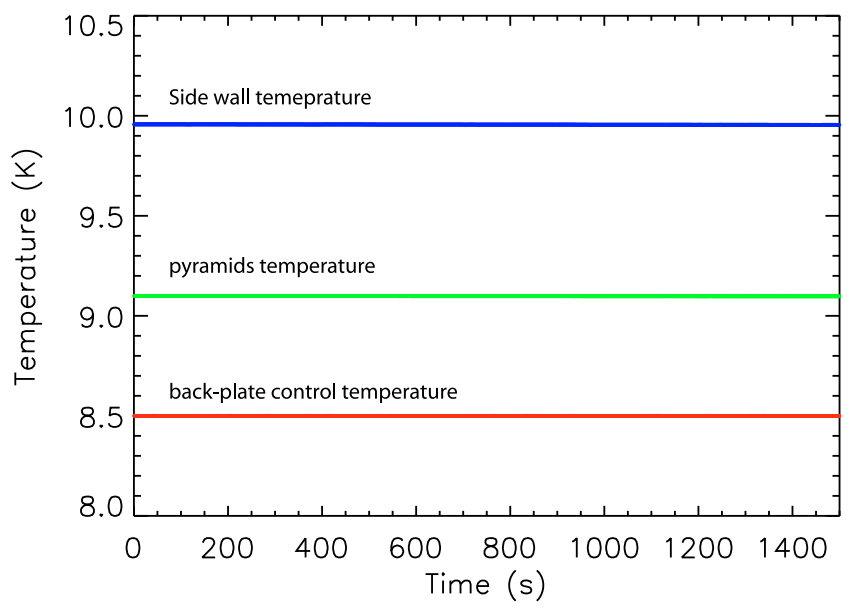

Fig. 3. Thirty minutes of data acquired during RCA28. Noise temperature and linearity tests are shown with $T_{\text {sky }}^{\text {ctrl }}$ in red, $T_{\text {sky }}$ in green, and $T_{\text {sky }}^{\text {side }}$ in blue. This represents the worst case of these differences. The stability of the temperatures with the values $T_{\text {sky }}^{\mathrm{ctrl}}=8.50000 \pm 0.00006$, $T_{\text {sky }}=9.0981 \pm 0.0004$, and $T_{\text {sky }}^{\text {side }}=9.9560 \pm 0.0007$ are also evident.

cavity was covered with Eccosorb pyramids to guarantee a return loss of about $-30 \mathrm{~dB}$. Details of the skyload are reported in Cuttaia (2005). Four temperature sensors were placed on the sky load, but only two cernox sensors were taken as reference for calibration. The first one was placed on the back plate of the sky load to measure the temperature of the PID control loop of the sky load, $T_{\text {sky }}^{\text {ctrl }}$ The second was placed on the Eccosorb pyramids inside the black body cavity and was assumed as the blackbody reference temperature, $T_{\text {sky }}$. The contribution to the effective emissivity due to the pyramids was estimated by Cuttaia (2005) to be 0.9956 for the $30 \mathrm{GHz}$ channel and 0.9979 for the $44 \mathrm{GHz}$ channel. The effective emissivity was calculated assuming the horn near field pattern and the emissivity of the material. In the case of the skyload side walls the effective emissivity is $4.29 \times 10^{-3}$ and $2.07 \times 10^{-3}$ for the $30 \mathrm{GHz}$ and $44 \mathrm{GHz}$ respectively. In the data analysis only the contribution of the pyramids was considered assuming its emissivity equal to 1 . Assuming the emissivities reported above and the temperatures as in Fig. 3, the approximation leads to an uncertainty in the brightness temperature of about $0.04 \mathrm{~K}$ and the same uncertainty in the noise temperature measurements.

Due to a failure in the sensor on the pyramids an analytical evaluation of $T_{\text {sky }}$ temperature from $T_{\text {sky }}^{\text {ctrl }}$ temperature was performed during the calibration of RCA24 and RCA27. The data are shown in Fig. 4. Although the data show a linear behavior, the differences between $T_{\text {sky }}^{\text {ctrl }}$ and $T_{\text {sky }}$ decrease as the
Table 3. Temperarture of the sky load pyramids. Quadratic fit coefficients.

\begin{tabular}{ccc}
\hline \hline & $30 \mathrm{GHz}$ & $44 \mathrm{GHz}$ \\
\hline$a$ & $0.9185 \pm 0.0006$ & $0.5430 \pm 0.008$ \\
$b$ & $0.9540 \pm 0.0001$ & $0.9795 \pm 0.0008$ \\
$c$ & $0.0008460 \pm 4.4 \times 10^{-6}$ & $0.000217 \pm 1.9 \times 10^{-5}$ \\
\hline
\end{tabular}
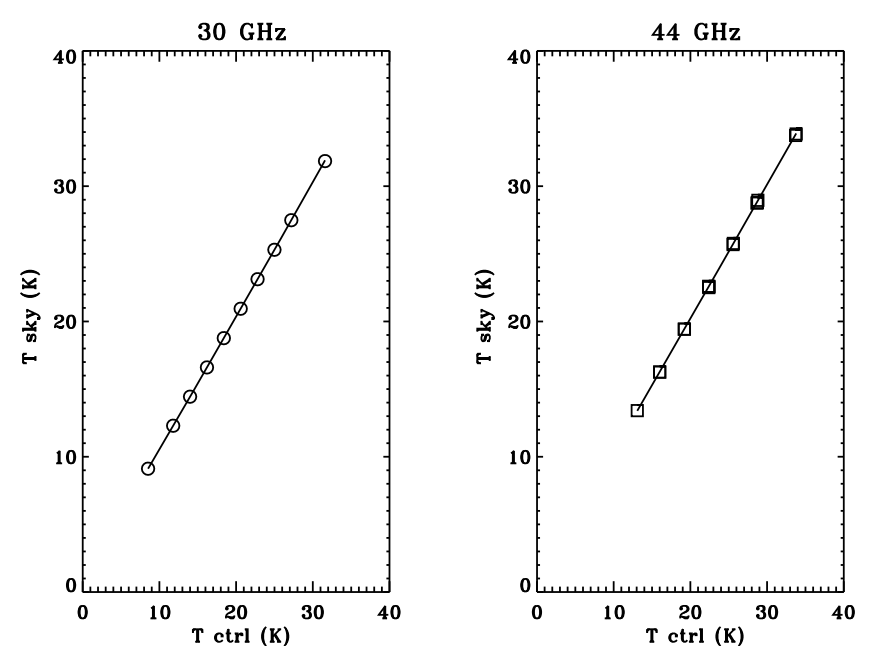

Fig. 4. $T_{\text {sky }}$ as a function of back plate controller skyload temperature $T_{\text {sky }}^{\text {ctrl }}$. The left plot refers to the $30 \mathrm{GHz}$ RCAs, based on RCA28 data (circles). The right plot refers to the $44 \mathrm{GHz}$ RCAs, based on RCA25 and RCA26 data (squares). The lines are the quadratic fit to the data.

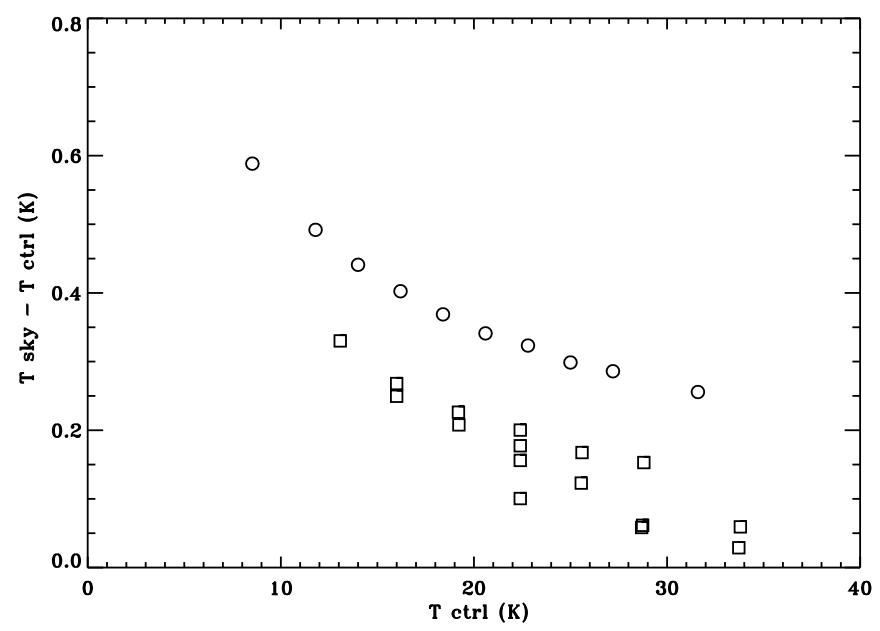

Fig. 5. Differences between $T_{\text {sky }}$ and $T_{\text {sky }}^{\text {ctrl }}$ as a function of $T_{\text {sky }}^{\text {ctrl }}$ showing the non-linear behavior of the difference. Circles are for $30 \mathrm{GHz}$ RCAs and squares for $44 \mathrm{GHz}$ RCAs.

temperature increases (see Fig. 5) as expected from the thermal behavior of the system, suggesting that a quadratic fit with $T_{\text {sky }}=a+b \cdot T_{\text {sky }}^{\mathrm{ctrl}}+c \cdot\left(T_{\mathrm{sky}}^{\mathrm{ctrl}}\right)^{2}$ is more representative. This quadratic fit was performed, and the coefficients are reported in Table 3.

\subsection{The cryofacility of the $70 \mathrm{GHz} R C A s$}

This cryofacility has the dimensions $1.6 \times 1.0 \times 0.3 \mathrm{~m}^{3}$. The facility has a layout similar to that at $30-44 \mathrm{GHz}$, although the $70 \mathrm{GHz}$ facility was designed to house two radiometer chains simultaneously (Fig. 7). 


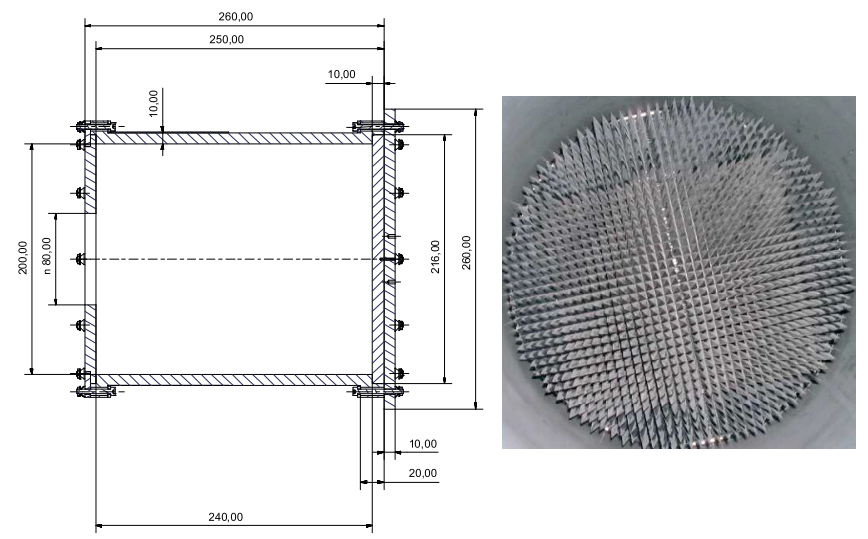

Fig. 6. Bologna design of the RCA sky load calibrator. The overall dimensions in $\mathrm{mm}$ are reported in the drawing on the left. The pyramids on the bottom of the skyload are clearly visible on the right picture.

The smaller dimensions of the feedhorns and front end modules and the decision to use two small dedicated sky loads directly in front of the horns allowed the cold part of the two RCAs under test to be contained in a volume similar to that of the 30 and $44 \mathrm{GHz}$ chamber. Temperature interfaces such as FEMs, sky load and reference load were coupled together by means of copper slabs and then connected to the $4 \mathrm{~K}$ and $20 \mathrm{~K}$ coolers. The FEMs were controlled at their nominal temperature of $20 \mathrm{~K}$; sky and reference loads were controlled in the range 10-25 K with a stability better than $10 \mathrm{mK}$; the back end modules were insulated from the chamber envelope by means of a supporting structure without temperature control, which was considered unnecessary.

\subsection{The skyloads at $70 \mathrm{GHz}$}

The design for the $70 \mathrm{GHz}$ RCA skyload was made by Ylinen Electronics. The basic design is shown in Fig. 8. The load configuration is a single folded conical structure in Eccosorb mounted in an aluminum housing. It is attached to a brass back plate. A single waveguide input is mounted through the back plate, providing the method of applying RF stimulus signals through the absorber for the RCA_SPR test (see Sect. 4.5). Load performances were measured over the whole $V$-band showing a return loss better than $-20 \mathrm{~dB}$. Two sensors were placed on the sky load, one at the controller stage, referred to as $T_{\text {ctrl }}$ and one inside the absorber, $T_{\text {sky. }}$ Although the temperature along the skyload was expected to be uniform due to its small dimensions, this was not the case: due to the cool down effects, the thermal junction between the temperature control and the load was not efficient as expected. A typical difference in temperature within 4-7 K was observed between the two thermometers. This cool-down effect was not predictable so that the sky load was considered as a relative instead of an absolute temperature reference.

\section{Methods and results}

\subsection{Functional tests}

Functional tests were performed at ambient and at cryo temperature. All the RCAs were biased with nominal values and the power consumption was verified. In addition, each phase switch was operated in the nominal mode to check its functionality. As an example the functional test performed at cryogenic temperature on RCA26 is shown in Fig. 9. The figure reports
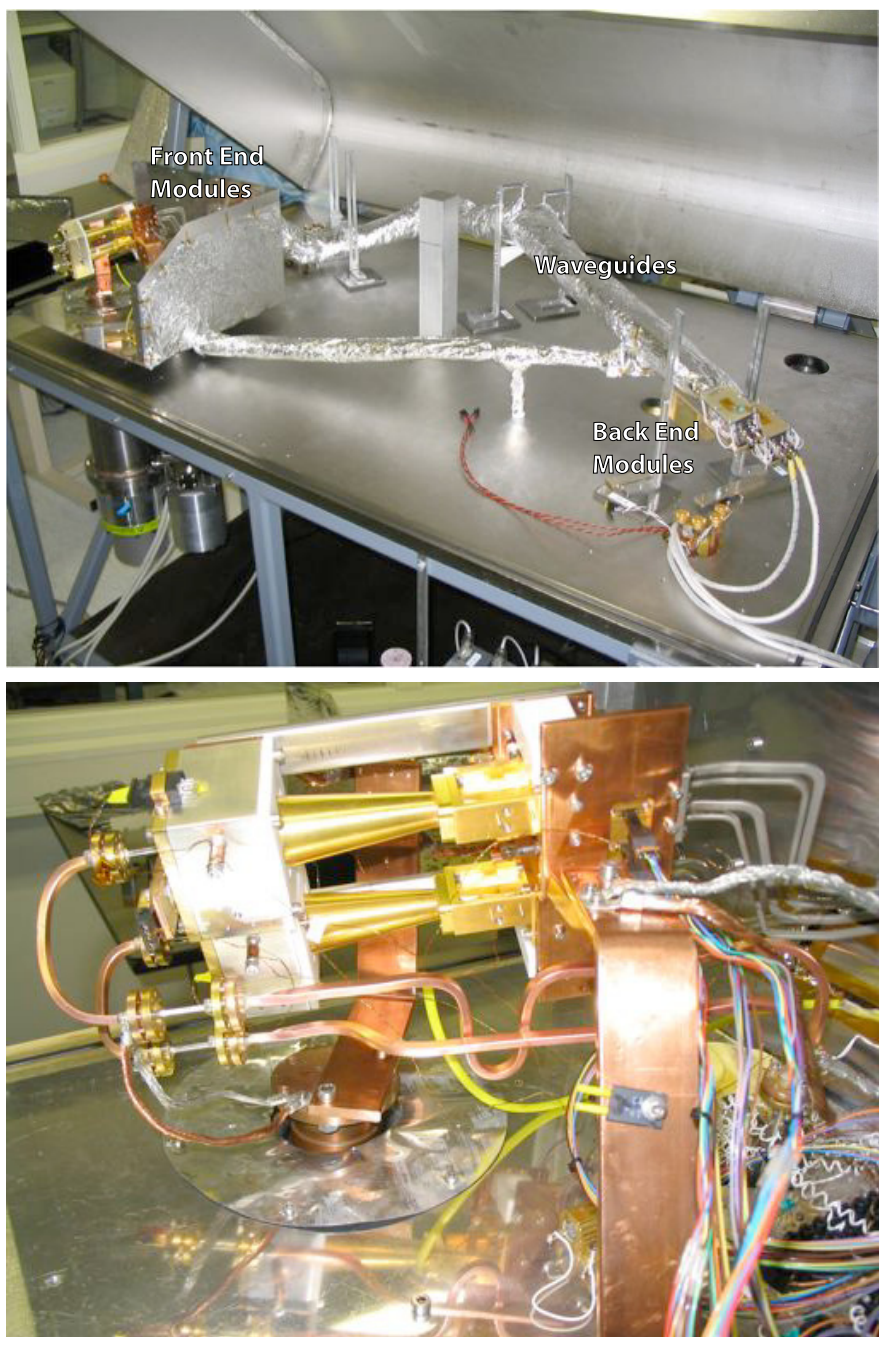

Fig. 7. Top: two $70 \mathrm{GHz}$ RCAs integrated in the Ylinen Electronics cryofacility. The two BEMs (on the right) are connected to the waveguides, here surrounded by aluminum mylar. On the left the shroud contains the two horns facing the "Ylinen" skyload at about $50 \mathrm{~K}$. Bottom: detail of the front end. The two FEMs and the pair of horns are facing the two skyload containers.

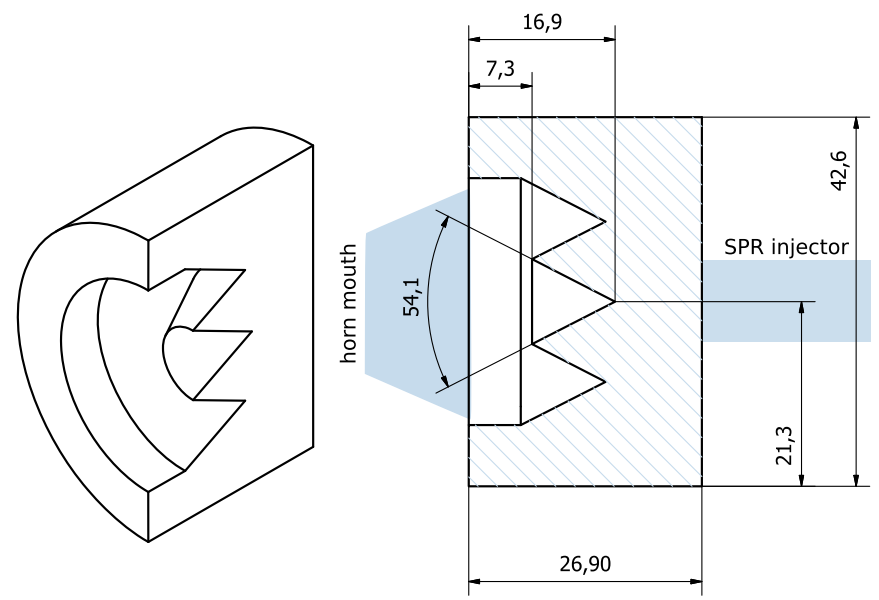

Fig. 8. Ylinen design of the RCA sky load calibrator. This design produced a load with $-20 \mathrm{~dB}$ of return loss over the whole bandwidth. The two light blue shaded regions represent the horn mouth at the left and the rectangular waveguide injector on the right. The absorber is enclosed in a metallic box except for the part facing the horn, which is closed with a Teflonßplate. 


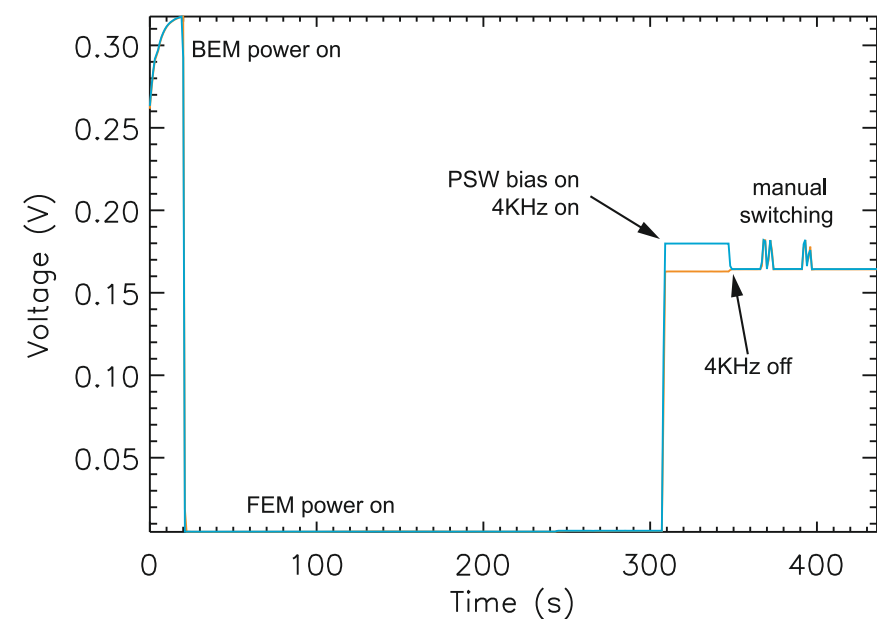

Fig. 9. Functional test performed at cryogenic temperature on RCA26. The two lines (orange and cyan) refer to the sky and load signals, when the $4 \mathrm{KHz}$ switching is activated. Outside the interval $310-350 \mathrm{~s}$ the two curves are indistinguishable.

the output voltage of the detector S-10 when the functional test is run: the BEM is switched on, the FEM is biased at nominal conditions and the fast $4 \mathrm{KHz}$ switching is activated on the phase switches. It is evident that most of the change in signal is experienced when the BEM is on and the phase switches are biased correctly. These functional tests were also used as a reference for further tests up to the satellite-level verification campaign, besides checking the functionality of the RCA to proceed with the calibration.

\subsection{Tuning}

Before tuning the RCA, the DAE was set-up to read the voltage from each detector of the BEM, $V_{\mathrm{BEM}}$, with appropriate resolution. The output signal from the DAE, $V_{\mathrm{DAE}}$, is given by

$V_{\mathrm{DAE}}=G^{\mathrm{DAE}} \cdot\left(V_{\mathrm{BEM}}-O_{\mathrm{DAE}}\right)$.

The DAE gain, $G^{\mathrm{DAE}}$, was set to ensure that the noise induced by the DAE did not influence the noise of the radiometric signal from the BEM detectors. The voltage offset, $O_{\mathrm{DAE}}$ was adjusted to guarantee that the output voltage signal lay well within the $[-2.5,+2.5]$ Volts range when the gain was set properly for the input temperature range. $G_{\mathrm{DAE}}$ and $O_{\mathrm{DAE}}$ were set for each of the four detectors and employed during all noise property tests.

The aim of the RCA tuning procedure (RCA_TUN) was to choose the best bias conditions for each FEM low noise amplifiers' (LNA) gate voltage and phase switch current. Each of the four LNAs in a $30 \mathrm{GHz}$ FEM consists of four amplification stages (five for the FEM at $44 \mathrm{GHz}$ ), each driven by the same drain voltage, $V_{\mathrm{d}}$. The gate voltage $V_{\mathrm{g} 1}$ biases the first amplification stage, while $V_{\mathrm{g} 2}$ biases the successive three (or four) stages. The phase switches are driven by two currents $\left(I_{1}\right.$ and $\left.I_{2}\right)$, biasing each diode. The currents determine the amount of attenuation by each diode and thus are adjusted to obtain the final overall radiometer balance.

The phase switches between the LNA and the second hybrid use the interconnection of two hybrid rings to improve the bandwidth and the matching with two Shunt PIN diodes. Depending on the polarization of the diodes the signal travels into a circuit, which can be $\lambda / 2$ longer, so that it is shifted by $180^{\circ}$. Details can be found in Hoyland (2004) and in Cuttaia et al. (2009). In

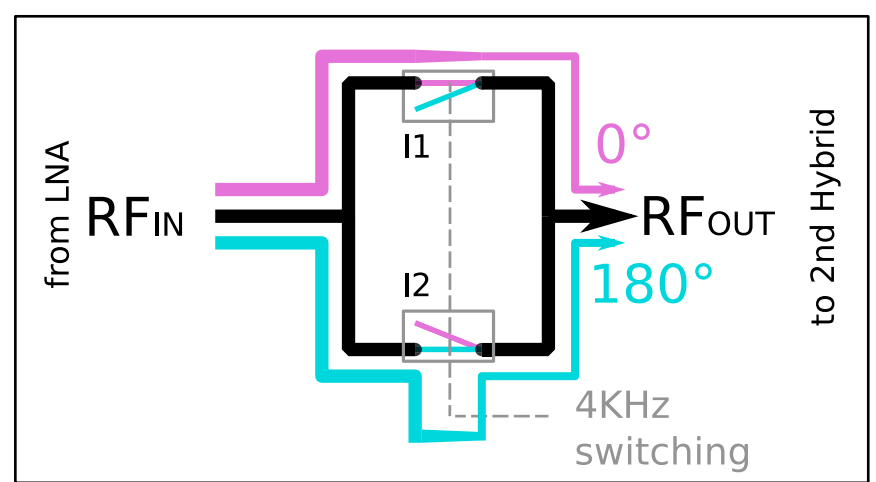

Fig. 10. Conceptual scheme of the phase switch integrated into the radiometers. Each phase switch is composed of two diodes commanded by the currents I1 and I2. They act as a on/off switch. Depending on the polarization state of the diodes the signal follows the magenta path or the cyan $\lambda / 2$ shifted path. The two currents at which the diodes are tuned determine the attenuation of each path, represented here by the different thickness. While the phase matching depends on the particular RF design, the amplitude matching depends on the $\left(I_{1}, I_{2}\right)$ bias supply of the diodes which is the goal of the phase switch tuning.

Fig. 10 we report the conceptual schematic diagram of the phase switch.

At 30 and $44 \mathrm{GHz}$ the phase switches were tuned with one radiometer leg switched off. In these conditions the signal entering each phase switch diode is the same, and the output signal at the DAE can be used directly to precisely balance the two states of the phase switch. Any differences in the sky and ref signal are caused only by the phase switches and not by other non-idealities of the radiometers, nor by different input target temperatures. The two currents were chosen to minimize the quadratic differences, $\delta^{\mathrm{PSW}}$, between odd and even samples of the signal (corresponding in Fig. 10 to the magenta and cyan path respectively). If for example the phase switch was tuned on the same leg as the amplifier M1, the following expression was minimized:

$\delta_{\mathrm{M} 1}^{\mathrm{PSW}}=\sqrt{\left(S_{00}^{\mathrm{o}}-S_{00}^{\mathrm{e}}\right)^{2}+\left(S_{01}^{\mathrm{o}}-S_{01}^{\mathrm{e}}\right)^{2}}$

where $S_{00}, S_{01}$ are the two DAE outputs related to the "M" half FEM. In this case o and e refer to odd and even signal samples. The same differences for the other phase switches, $\delta_{\mathrm{M} 2}^{\mathrm{PSW}}$, $\delta_{\mathrm{S} 1}^{\mathrm{PSW}}$, and $\delta_{\mathrm{S} 2}^{\mathrm{PSW}}$ were calculated in the same way. The $I_{1}$ and $I_{2}$ were varied around the best value obtained during the FEM stand-alone tests (Davis et al. 2009). The phase switches of the $70 \mathrm{GHz}$ RCAs were not tuned. To reduce the transient, the phase switches were always biased at the maximum allowable current.

The front-end LNAs were tuned for noise temperature performance, $T_{n}$, and isolation, $I$. For each channel $T_{n}$ and $I$ were measured as a function of the gate voltages $V_{\mathrm{g} 1}$ and $V_{\mathrm{g} 2}$.

Firstly the minimum noise conditions were found by varying $V_{\mathrm{g} 1}$ while keeping $V_{\mathrm{g} 2}$ and $V_{\mathrm{d}}$ fixed. The noise temperature was measured with the $Y$-factor method (see Appendix A for the details of this method). Because only relative estimates of Tn are relevant for tuning purposes, we did not correct for the effect of non-linearity in the 30 and $44 \mathrm{GHz}$ RCAs.

Once the optimum $V_{\mathrm{g} 1}$ was determined, the optimum $V_{\mathrm{g} 2}$ was found by maximizing the isolation, $I$,

$I=\frac{\Delta V_{\text {sky }}-G_{0} \cdot \Delta T_{\text {sky }}}{\left(\Delta V_{\text {ref }}-G_{0} \cdot \Delta T_{\text {sky }}\right)+\Delta V_{\text {sky }}}$ 
measured by varying $T_{\text {ref }}$ with $T_{\text {sky }}$ kept fixed. The term $-G_{0}$. $\Delta T_{\text {sky }}$ is a correction factor for any unwanted variation of $T_{\text {sky }}$ originating chiefly from thermal non-ideality of the cryofacility.

At $70 \mathrm{GHz}$ the best working conditions were found by measuring the noise temperature and the isolation as a function of the gate voltages $V_{\mathrm{g} 1}$ and $V_{\mathrm{g} 2}$, but with a slightly different approach mainly for schedule reasons to that of the lower frequency chains. The procedure required two different temperatures for the reference load (about $10 \mathrm{~K}$ in the low state and about $20 \mathrm{~K}$ in the high state) and $V_{\mathrm{g} 1}, V_{\mathrm{g} 2}$, and $V_{\mathrm{d}}$ were varied independently on the half FEM. Then the procedure was repeated with both FEM legs switched on. The noise temperature was measured with the $Y$-factor method from the signals coming from the two temperature states, and the isolation was calculated with Eq. (16).

Although the bias parameters found during the RCA tuning are the optimal ones, we found that different electrical and cryogenic conditions induce uncertainties in the bias values. This is due to the different grounding and the impact of the thermal gradient along the bias cables. To overcome this problem, tuning verification campaigns are planned at LFI integrated level and inflight. In both cases the RCA bias values have been assumed as reference values.

\subsection{Basic properties}

The basic properties of the radiometers, namely noise temperature, isolation, gain, and linearity were obtained in a single test.

The RCA_LIS test was performed varying $T_{\text {sky }}$ (and subsequently $T_{\text {ref }}$ ) in steps while keeping the $T_{\text {ref }}$ (and subsequently $T_{\text {sky }}$ ) constant. In Fig. 11 we give the temperature range spanned during the tests. Due to the different thermal behavior of each RCA the range was not the same for all the chains. The brightness temperature was calculated from temperature sensors located in the external calibrators (both sky and reference) and the output voltages of the four detectors were recorded. The main uncertainty was in the determination of the actual brightness temperature seen by the radiometer. At 30 and $44 \mathrm{GHz}$ the brightness temperature of the skyload was derived from the thermometer located inside the pyramids, from where the main thermal noise emission originated. For $70 \mathrm{GHz}$ both the backplate thermometer and the absorber thermometer were used to derive the brightness temperature. However, the backplate and the absorber temperatures were found to introduce a significant systematic error in the reconstructed physical temperature of the load, as explained in Sect. 3.4. It was decided to calibrate the $70 \mathrm{GHz}$ RCAs using the reference load steps instead.

We denote here for simplicity the value of either $V_{\text {out }}^{\text {sky }}$ or $V_{\text {out }}^{\text {ref }}$ with $V_{\text {out }}$ in Eqs. (5) and (8), alternatively $T_{\text {sky }}$ or $T_{\text {ref }}$ with $T_{\text {in }}$, and the corresponding $\widetilde{T}_{\mathrm{N}}^{\text {sky }}$ or $\widetilde{T}_{\mathrm{N}}^{\text {ref }}$ with $T_{\text {sys }}$. With $G$ we denote the corresponding total gain.

For a perfectly linear radiometer the output signal can be written as

$V_{\text {out }}=G \cdot\left(T_{\text {in }}+T_{\text {sys }}\right)$,

and the gain and system temperature can be calculated by measuring the output voltage for only two different values of the input temperature ( $Y$-factor method). This was indeed the case for the $70 \mathrm{GHz}$ RCAs. For the 30 and $44 \mathrm{GHz}$ RCAs the determination of the basic properties was complicated by a significant non-linear component in the response of the 30 and $44 \mathrm{GHz}$ RCAs. This was discovered during the previous qualification campaign and has been well characterized during these flight

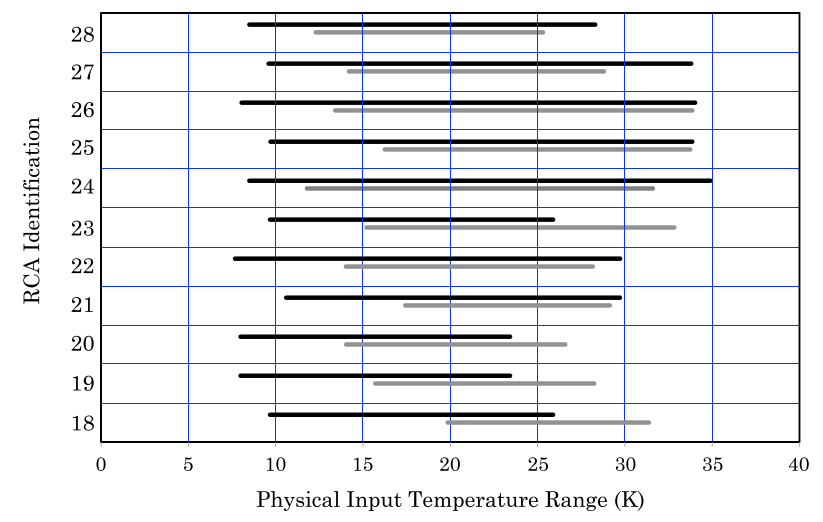

Fig. 11. Physical temperature ranges for the RCA_LIS tests. Black lines refer to the reference load temperature steps. Gray lines refer to the skyload temperature steps.

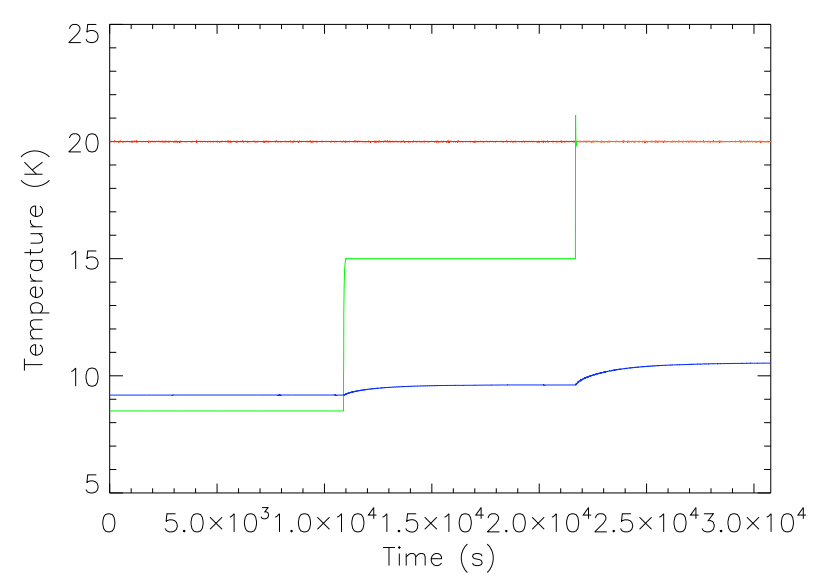

Fig. 12. Typical temperature behavior of sky load (blue), reference load (green) and FEM body (red) during the RCA_STn test. The temperature stability is better than $1 \mathrm{mK}$.

model RCA tests. The non-linearity effects in LFI are discussed in Mennella et al. (2009) together with its impact on the science performances. For a radiometer with compression effects the radiometer gain, $G$, is a function of total input temperature, $T=T_{\text {in }}+T_{\text {sys }}$ and is given by

$V_{\text {out }}=G(T) \cdot\left(T_{\text {in }}+T_{\text {sys }}\right)$.

Particular care was required in the determination of the noise temperature of the 30 and $44 \mathrm{GHz}$ RCAs. To overcome the problem, the application of four different types of fit were performed:

1. Linear fit. This fit was always calculated as a reference, even for non-linear behavior of the radiometer, so that

$V_{\text {out }}=G_{\text {lin }} \cdot T$.

The linear gain, $G_{\text {lin }}$, and the noise temperature were derived. The fit was applied to all available data, not only to the two temperature steps as in the $Y$-factor method, to reduce the uncertainties for the linear $70 \mathrm{GHz}$ RCAs and to evaluate the non-linearity of the 30 and $44 \mathrm{GHz}$ chains.

2. Parabolic fit. This was applied to understand the effect of the non-linearity, for the evaluation of which the quadratic fit is the simplest way. The output of the fit were the three coefficients from the equation

$V_{\text {out }}=a_{0}+a_{1} T+a_{2} T^{2}$. 
Table 4. Receiver basic properties.

\begin{tabular}{lrrrr}
\hline \hline & \multicolumn{4}{c}{ ISOL (dB) } \\
& M-00 & M-01 & \multicolumn{1}{c}{ S-10 } & S-11 \\
\hline RCA18 & -13.5 & -13.0 & -11.1 & -10.9 \\
RCA19 & -15.5 & -15.9 & -15.3 & -13.9 \\
RCA20 & -15.8 & -15.9 & -12.7 & -14.2 \\
RCA21 & -13.0 & -12.4 & -10.1 & -10.4 \\
RCA22 & -12.8 & -11.1 & -12.4 & -11.3 \\
RCA23 & -12.6 & -11.8 & -13.3 & -14.3 \\
RCA24 & -11.7 & -12.3 & -10.4 & -10.5 \\
& $(-13.3)$ & $(-13.6)$ & $(11.6)$ & $(-11.9)$ \\
RCA25 & -10.8 & -10.7 & -12.0 & -11.5 \\
& $(-14.6)$ & $(-14.4)$ & $(-15.5)$ & $(-14.5)$ \\
RCA26 & -10.8 & -11.9 & -13.7 & -13.7 \\
& $(-9.7)$ & $(-10.4)$ & $(-13.9)$ & $(-14.0)$ \\
RCA27 & -13.0 & -12.8 & -14.7 & -14.6 \\
& $(-11.2)$ & $(-11.0)$ & $(-11.7)$ & $(-11.8)$ \\
RCA28 & -10.9 & -10.3 & -10.3 & -10.5 \\
& $(-11.6)$ & $(-11.2)$ & $(-12.0)$ & $(-12.2)$ \\
\hline
\end{tabular}

Notes. Isolation in $\mathrm{dB}$. The values found during the $V_{g 2}$ tuning are also reported in brackets for comparison.

In this case the noise temperature was employed as the solution of the equation $a_{2} T_{\text {sys }}^{2}+a_{1} T_{\text {sys }}+a_{0}=0$.

3. Inverse parabolic fit. This was used because the noise temperature was directly derived from

$T=a_{0}+a_{1} V_{\text {out }}+a_{2} V_{\text {out }}^{2}$,

where $T_{\text {sys }}=a_{0}$.

4. Gain model fit. A new gain model was developed based on the results of Daywitt (1989), modified for the LFI (see Appendix B). The total power output voltage was written as

$V_{\text {out }}=\left[\frac{G_{0}}{1+b \cdot G_{0} \cdot\left(T_{\mathrm{in}}+T_{\mathrm{sys}}\right)}\right] \cdot\left(T_{\mathrm{in}}+T_{\mathrm{sys}}\right)$,

where $G_{0}$ is the total gain in the case of a linear radiometer, $b$ is the linear coefficient $(b=0$ in the linear case, $b=\infty$ for complete saturation, i.e. $G(T)=0)$.

The values obtained for gain, linearity and noise temperature are reported in Table 5. The isolation values were calculated with Eq. (16) based on all possible combinations of temperature variation on the reference load. The results are given in Table 4, where the values obtained during the tuning of the $V_{\mathrm{g} 2}$ are also reported for the 30 and $44 \mathrm{GHz}$ RCAs. While at $30 \mathrm{GHz}$ the differences are due mainly to the reference load thermal model applied in this case, for the $44 \mathrm{GHz}$ RCAs the differences are dominated by the gain used to compensate for thermal coupling in Eq. (16).

\subsection{Noise properties}

Radiometer noise properties were derived from the RCA_STn test. This test consisted of acquiring data under stable thermal conditions for at least three hours. Then the temperature of the loads were changed to measure the noise properties at different sky and reference target temperatures. As expected, the best $1 / f$ conditions were found when the difference between the sky and reference load temperatures was minimal. This occurred at the first and last step as seen in Fig. 12, which represents a typical
Table 5. Receiver basic properties: gain, noise temperature, and linearity.

\begin{tabular}{llrrrr}
\hline \hline \multicolumn{6}{c}{ GAIN $(\mathrm{V} / \mathrm{K}), T_{\mathrm{n}}(\mathrm{K})$, AND LIN } \\
& & $\mathrm{M}-00$ & $\mathrm{M}-01$ & $\mathrm{~S}-10$ & $\mathrm{~S}-11$ \\
\hline RCA18 & $G_{0}$ & 0.0173 & 0.0195 & 0.0147 & 0.0143 \\
& $T_{\mathrm{n}}$ & 36.0 & 36.1 & 33.9 & 35.1 \\
$\mathrm{RCA19}$ & $G_{0}$ & 0.0161 & 0.0174 & 0.0176 & 0.0196 \\
& $T_{\mathrm{n}}$ & 33.1 & 31.5 & 32.2 & 33.6 \\
RCA20 & $G_{0}$ & 0.0186 & 0.0164 & 0.0161 & 0.0165 \\
& $T_{\mathrm{n}}$ & 35.2 & 34.2 & 36.9 & 35.0 \\
RCA21 & $G_{0}$ & 0.0161 & 0.0154 & 0.0119 & 0.0114 \\
& $T_{\mathrm{n}}$ & 27.3 & 28.4 & 34.4 & 36.4 \\
RCA22 & $G_{0}$ & 0.0197 & 0.0174 & 0.0165 & 0.0163 \\
& $T_{\mathrm{n}}$ & 30.9 & 30.3 & 30.3 & 31.8 \\
RCA23 & $G_{0}$ & 0.0149 & 0.0171 & 0.0271 & 0.0185 \\
& $T_{\mathrm{n}}$ & 35.9 & 34.1 & 33.9 & 31.1 \\
RCA24 & $G_{0}$ & 0.0048 & 0.0044 & 0.0062 & 0.0062 \\
& $T_{\mathrm{n}}$ & 15.5 & 15.3 & 15.8 & 15.8 \\
& $b$ & 1.79 & 1.49 & 1.44 & 1.45 \\
RCA25 & $G_{0}$ & 0.0086 & 0.0085 & 0.0079 & 0.0071 \\
& $T_{\mathrm{n}}$ & 17.5 & 17.9 & 18.6 & 18.4 \\
& $b$ & 1.22 & 1.17 & 0.80 & 1.01 \\
RCA26 & $G_{0}$ & 0.0052 & 0.0067 & 0.0075 & 0.0082 \\
& $T_{\mathrm{n}}$ & 18.4 & 17.4 & 16.8 & 16.5 \\
& $b$ & 1.09 & 1.42 & 0.94 & 1.22 \\
RCA27 & $G_{0}$ & 0.0723 & 0.0774 & 0.0664 & 0.0562 \\
& $T_{\mathrm{n}}$ & 12.1 & 11.9 & 13.0 & 12.5 \\
& $b$ & 0.12 & 0.12 & 0.13 & 0.14 \\
RCA28 & $G_{0}$ & 0.0621 & 0.0839 & 0.0607 & 0.0518 \\
& $T_{\mathrm{n}}$ & 10.6 & 10.3 & 9.9 & 9.8 \\
& $b$ & 0.19 & 0.16 & 0.19 & 0.20 \\
\hline & & & & &
\end{tabular}

Notes. For the $70 \mathrm{GHz}$ RCAs, the gain, $G_{0}$, and the noise temperature, $T_{n}$, were derived from the linear fit, and the linearity coefficient is not reported. For 30 and $44 \mathrm{GHz}, G_{0}, T_{n}$, and the linearity coefficient, $b$, were derived from the gain model-fit.

temperature profile of the test. The amplitude spectral density was calculated for each output diode. The $1 / f$ component (knee frequency, $f_{\mathrm{k}}$, and slope, $\alpha$ ), the white noise plateau and the gain modulation factor, $r$, were derived. At $70 \mathrm{GHz}$ the $1 / f$ spectrum was clearly dominated by thermal instabilities of the BEM which was not controlled in temperature. There the measured knee frequency values were over-estimated, while at 30 and $44 \mathrm{GHz}$ the cryofacility was sufficiently stable to characterize the $1 / f$ performances of the radiometers. From the white noise and DC level the effective bandwidth was calculated as

$\beta=K^{2} \cdot \frac{V_{\text {out }}^{2}}{\Delta V_{\text {out }}^{2} \cdot \tau}$

where $\Delta V_{\text {out }}$ is the white noise level, $V_{\text {out }}$ is the DC level, $\tau$ the integration time, and $K=1$ for a single detector total power in the unswitched condition, $K=\sqrt{2}$ for a single detector total power in the switched condition, $K=2$ for a single detector differenced data, $K=\sqrt{2}$ for double-diode differenced data. This formula does not include the non-linearity effects that are discussed in detail by Mennella et al. (2009). The overall noise performances of all eleven RCAs are reported in Table 6, while Fig. 13 shows the typical amplitude spectral density of the noise for each frequency channel.

Apart from $1 / f$ noise and white noise, spikes were observed in all RCAs: at $70 \mathrm{GHz}$ they were caused by the electrical interaction between the two DAE units, which were slightly unsynchronised; at 30 and $44 \mathrm{GHz}$ they were due to the housekeeping 

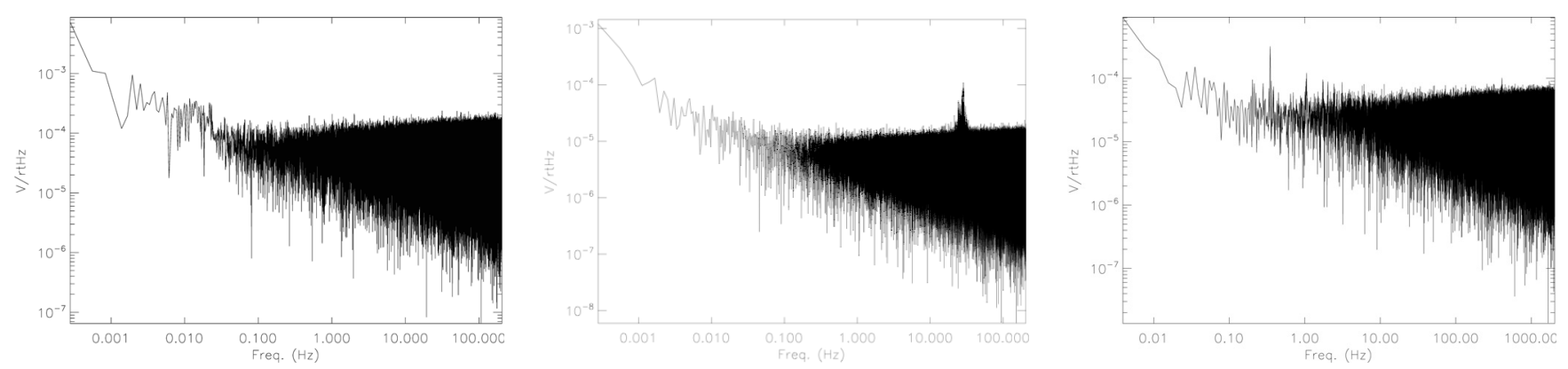

Fig. 13. Log-log plot of the amplitude spectral density of the differential detector output noise. The left plot refers to the RCA27M- 00 detector with the sky and the reference loads both at $20 \mathrm{~K}$; the plot in the center refers to the RCA26M-00 detector with sky and reference loads at $8 \mathrm{~K}$ and $13 \mathrm{~K}$ respectively; the plot on the right refers to the RCA23S-11 with the sky and reference loads at $15 \mathrm{~K}$ and $9 \mathrm{~K}$ respectively.

acquisition system. Because it was clear that the spikes were always due to the test setup and not to the radiometers themselves, the spikes were not considered critical at this stage, even if they showed up in frequency and amplitude.

As an example of the dependence of the noise performance on the temperature the antenna temperature pairs used during the the tests of the RCA28 are reported in Table 7. These temperatures were calculated with the coefficients reported in Tables 2 and 3 of Sect. 3.1 with the physical temperatures converted into antenna temperatures. The differences between the $T_{\text {ref }}$ and the $T_{\text {sky }}$ were calculated for each arm of the radiometer. The resulting $1 / f$ knee frequency, the slope of the $1 / f$ spectra, and the gain modulation factor, $r$, are reported in Fig. 14 as a function of the temperature differences. It is evident from these plots that the knee frequency is increasing with the temperature difference, as expected. Moreover, the gain modulation factor is approaching unity as the input temperature difference becomes zero, which agrees with Eq. (4). The slope, $\alpha$, does not show any correlation with the temperature differences, because it depends on the amplifiers rather than on the $\left(T_{\text {ref }}-T_{\text {sky }}\right)$. This behavior was also found in the other RCAs.

\subsection{Bandpass}

A dedicated end-to-end spectral response test, RCA_SPR, was designed and carried out to measure radiometer RF bandshape in operational conditions, i.e., on the integrated RCA with the front-end at the cryogenic temperature. An external RF source was used to inject a monochromatic signal sweeping through the band into the sky horn. Then the DC output of the radiometer was recorded as a function of the input frequency, giving the relative overall RCA gain-shape, $G_{\mathrm{spr}}(v)$. The equivalent bandwidth was calculated with

$\beta_{\mathrm{spr}}=\frac{\left(\int G_{\mathrm{spr}}(v) \mathrm{d} v\right)^{2}}{\int G_{\mathrm{spr}}(v)^{2} \mathrm{~d} v}$.

Different setup configurations were used. At $70 \mathrm{GHz}$ the RF signal was directly injected into the sky horn. The input signal was varied by 50 points from $57.5 \mathrm{GHz}$ to $82.5 \mathrm{GHz}$. At $30 \mathrm{GHz}$ and $44 \mathrm{GHz}$ the RF signal was injected into the sky horn after a reflection on the sky load absorber's pyramids, scanning in frequency from $26.5 \mathrm{GHz}$ to $40 \mathrm{GHz}$ in 271 points and from $33 \mathrm{GHz}$ to $50 \mathrm{GHz}$ in 341 points. The flexible waveguides WR28 and WR22 were used to reach the skyload for the 30 and $44 \mathrm{GHz}$ RCAs (Fig. 15). The input signal was not calibrated in power because only a relative band shape measurement was required. The stability of the signal was ensured by the use of a synthesized sweeper generator guaranteeing the stability of the output
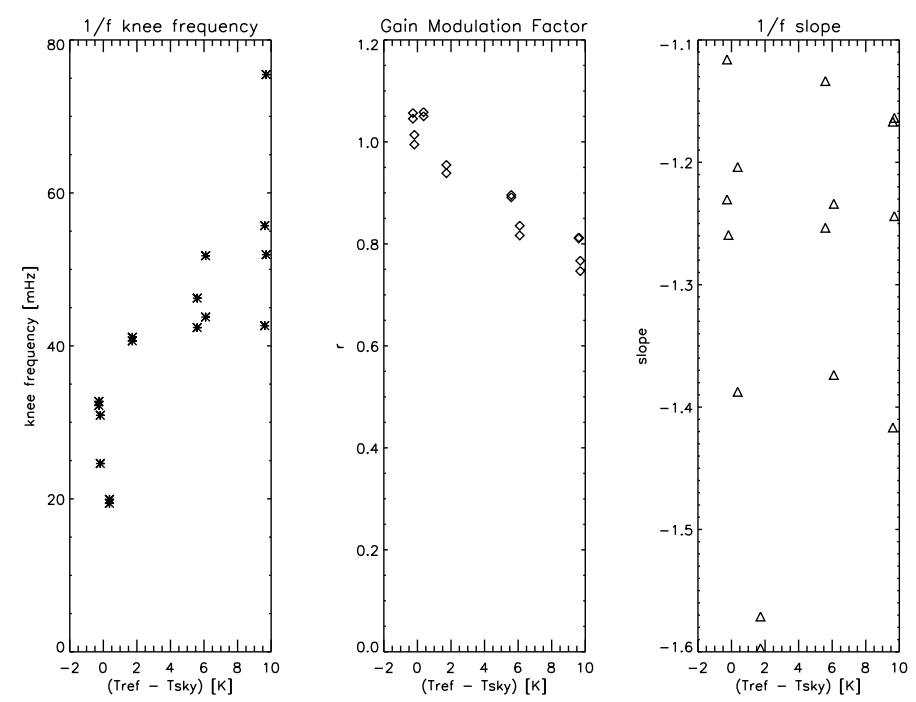

Fig. 14. $1 / f$ knee frequency (asterisk on the left), gain modulation factor (diamonds in the center), and the slope of the $1 / f$ spectrum (triangles on the right) as a function of $\left(T_{\text {ref }}-T_{\text {sky }}\right)$. Sixteen points (four pairs for each detector) were reported. The spread of knee frequency values is due to the intrinsic difficulty of fitting the lower part of the power spectral density.

within $10 \%$. The attenuation curve of the waveguide carrying the signal from the sweeper to the injector was treated as a rectangular standard waveguide with losses during the data analysis.

All RCA bandshapes were measured, but for the two $30 \mathrm{GHz}$ RCAs only half a radiometer was successfully tested due to a setup problem that appeared when the RCAs were cooled down. For schedule reasons it was not possible to repeat the test at the operational temperature, and only a check at the warm temperature was performed. This warm test was not used for calibration due to different dynamic range, amplifier behavior, and bias conditions. Results are reported in Table 8 and plots of all the measurements in Figs. 16-18. All curves reported in the plots are normalized to the area so that

$G_{\mathrm{spr}}^{\mathrm{n}}(v)=\frac{G_{\mathrm{spr}}(v)}{\int G_{\mathrm{spr}}(v) \mathrm{d} v}$

The bandshape is mainly determined by the filter located inside each BEM, whose frequency response is independent of the tuning of the FEM amplifiers. The dependence of the bandshape on the amplifier biases has been checked on the $30 \mathrm{GHz}$ radiometers (De Nardo 2008), showing that at first order the response remains unchanged. A similar situation occurs on the RCAs at 

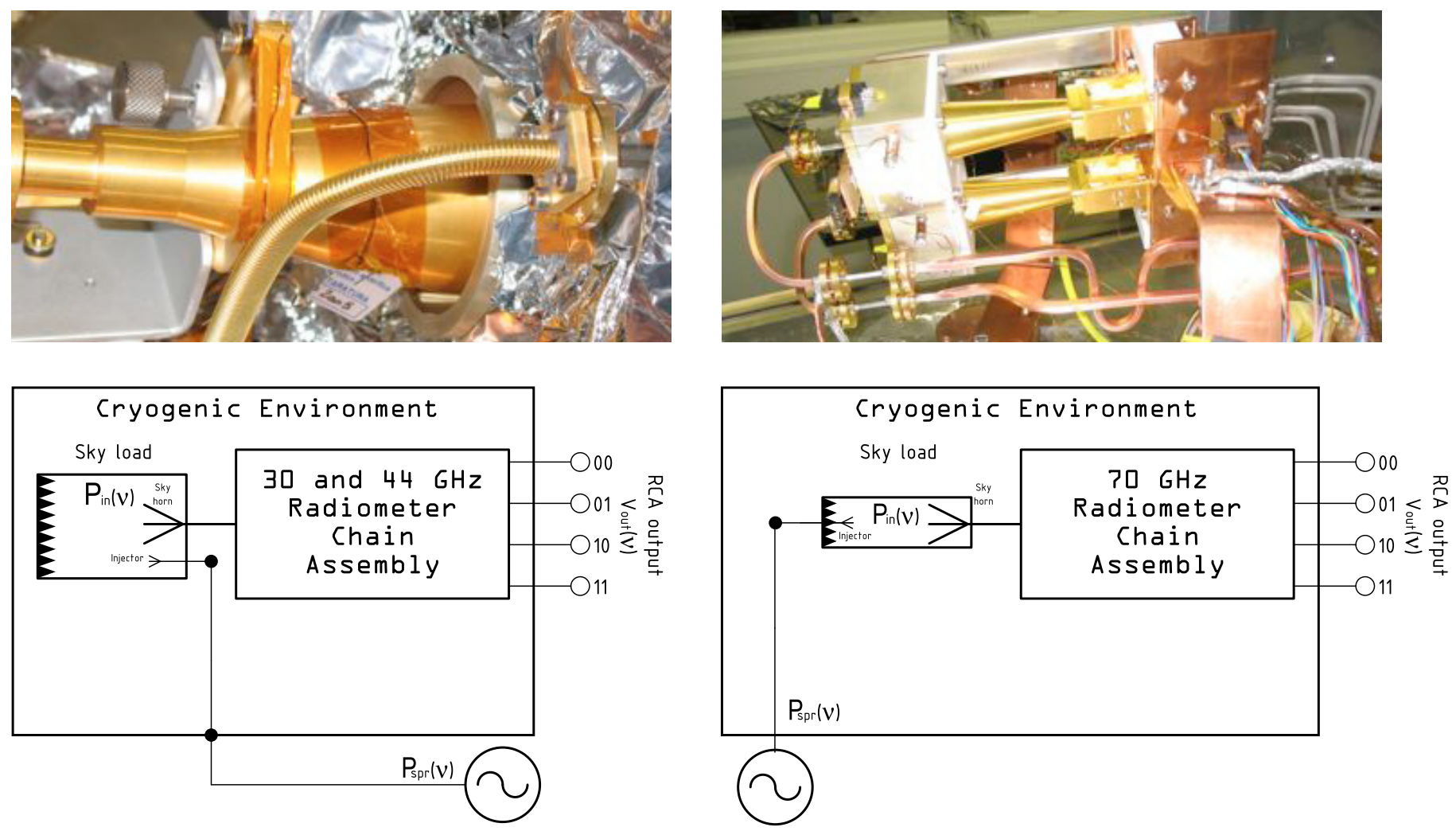

Fig. 15. Setup of the RCA_SPR tests. The picture and the sketch on the left report the test setup of the 30 and 44 GHz RCAs. The flexible waveguide is clearly visible on the picture on the side of the horn. The picture and the sketch on the right report the setup of the $70 \mathrm{GHz}$ RCAs. There the signal was injected infront of the horn through the sky load, and copper rigid waveguides were used to carry the signal form the generator to the RCAs.

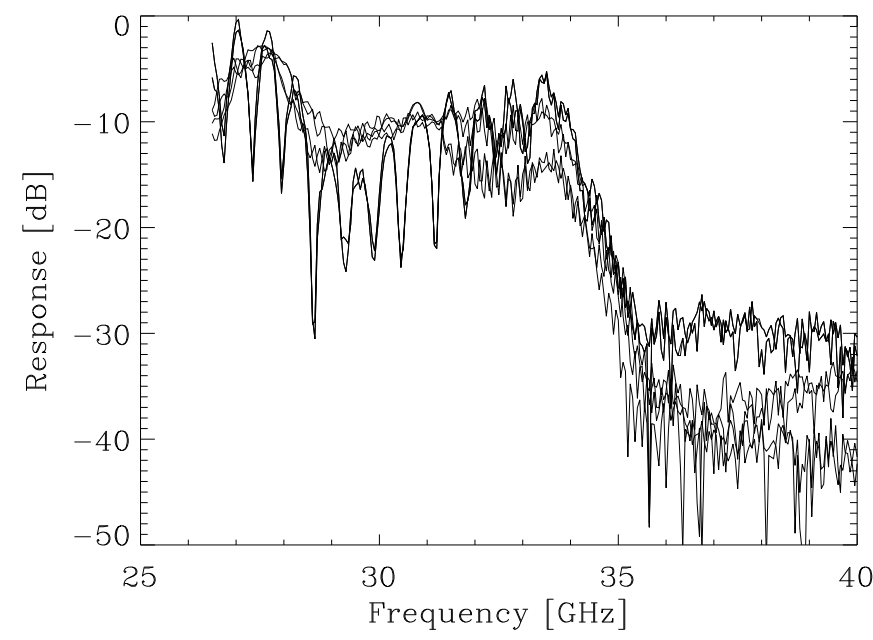

Fig. 16. Measured relative gain function (bandpass) of the 8 detectors at $30 \mathrm{GHz}$. The curves that show big ripples are those caused by the setup problem (see text). The bandpasses are normalized to the area as explained in the text.

$44 \mathrm{GHz}$ and $70 \mathrm{GHz}$, where the tuning has second order effects on the overall frequency response.

\subsection{Susceptibility}

Any variation in physical temperature of the RCA, $T_{\text {phys }}$, will produce a variation of the output signal that mimics the variation of the input temperature, $T_{\text {sky }}$, so that

$\delta T_{\text {sky }}=T_{\mathrm{f}} \cdot \delta T_{\text {phys }}$,

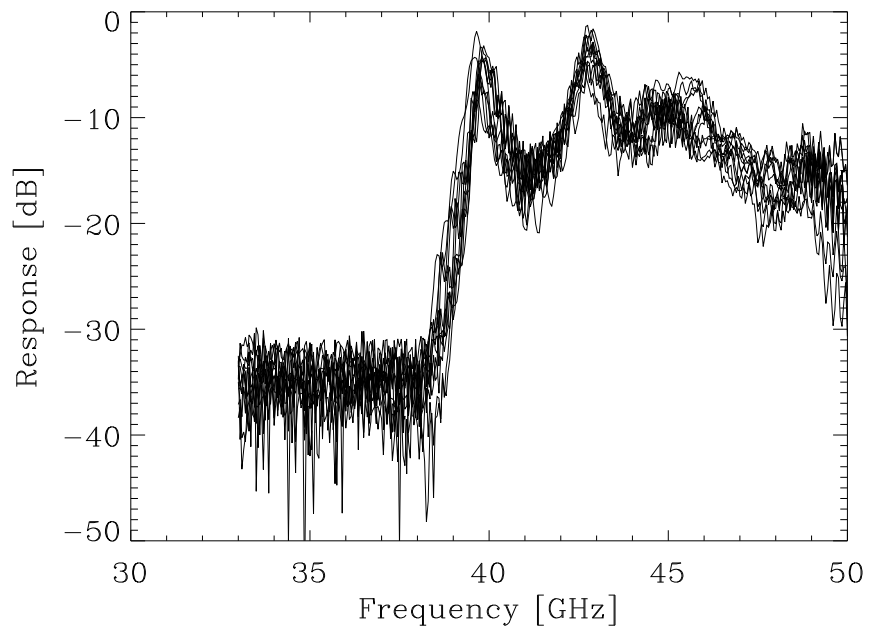

Fig. 17. Measured relative gain function (bandpass) of all 12 detectors at $44 \mathrm{GHz}$. The bandpasses are normalized to the area as explained in the text.

where $T_{\mathrm{f}}$ is the transfer function. A controlled variation of FEM temperature was imposed to calculate the transfer function of the front end modules, $T_{\mathrm{f}}^{\mathrm{FEM}}$. This was done for all RCAs and all detectors. The chief results are given in Table 9, while the details of the applied method and of the measurements are reported by Terenzi et al. (2009c).

The susceptibility of the radiometer signal to temperature variations in the BEM and 3rd V-groove were measured only for the 30 and $44 \mathrm{GHz}$ chains, because at $70 \mathrm{GHz}$ it was not possible to control the temperatures of these interfaces in their cryofacility. Here we report on the BEM susceptivity tests 
Table 6. Receiver noise properties: $1 / f$ knee frequency, slope, and $r$ factor.

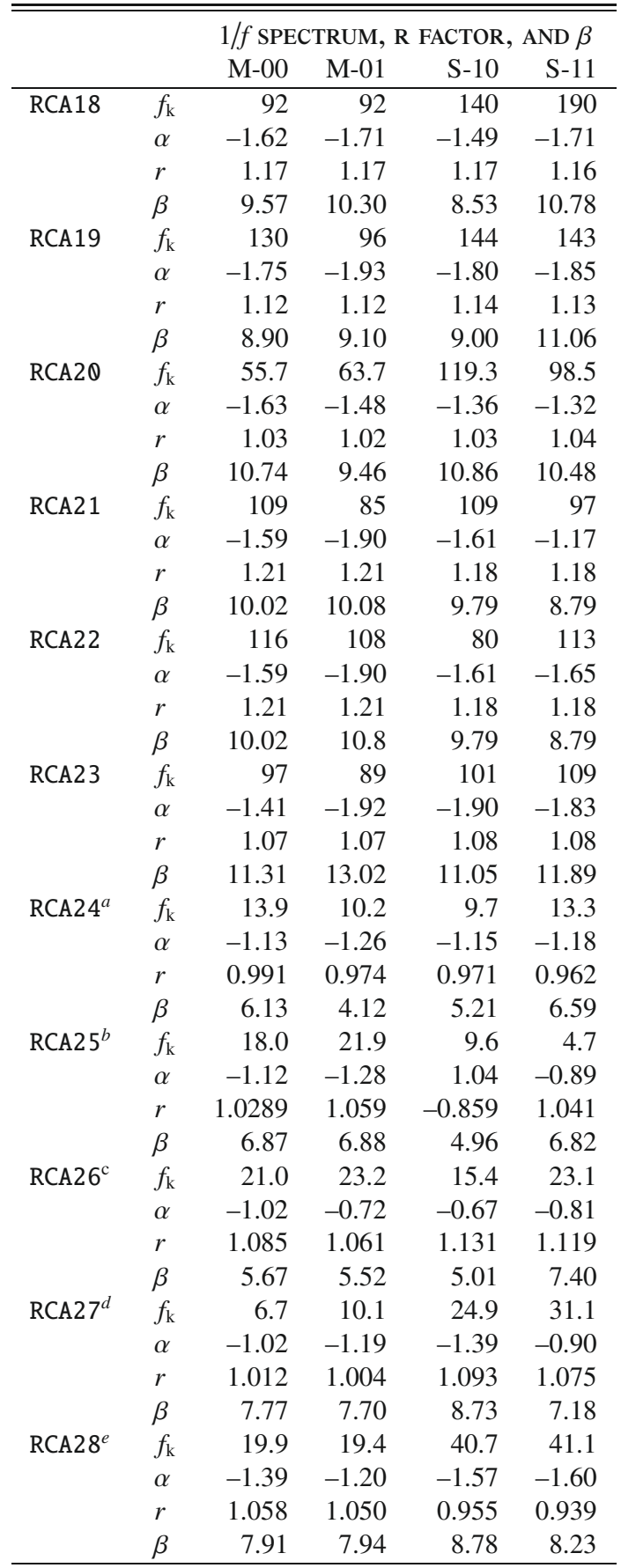

Notes. Data were taken setting the temperature of the loads at the lowest possible values. The $70 \mathrm{GHz} 1 / f$ knee frequencies are dominated by the thermal instabilities of the cryochamber. ${ }^{(a)} T_{\text {ref }}=8.5 \mathrm{~K} ; T_{\text {sky }}=8.5 \mathrm{~K}$. ${ }^{(b)} T_{\text {ref }}=8.0 \mathrm{~K} ; T_{\text {sky }}=10.5 \mathrm{~K} .{ }^{(c)} T_{\text {ref }}=8.0 \mathrm{~K} ; T_{\text {sky }}=13.0 \mathrm{~K} .{ }^{(d)} T_{\text {ref }}=$ $9.5 \mathrm{~K} ; T_{\text {sky }}=12.8 \mathrm{~K} .{ }^{(e)} T_{\text {ref }}=8.6 \mathrm{~K} ; T_{\text {sky }}=8.5 \mathrm{~K}$.

only: it is the prominent radiometric effect between both, because the diodes are thermally attached to the BEM body. The total power output voltage on each BEM detector can then be expressed by modifying Eq. (18)

$V_{\text {out }}=G_{\text {tot }}\left(T_{0}^{\text {bem }}\right) \cdot\left[1+T_{\mathrm{f}} \cdot\left(T^{\text {bem }}-T_{0}^{\text {bem }}\right)\right] \cdot\left(T_{\text {in }}+T_{\text {sys }}\right)$.

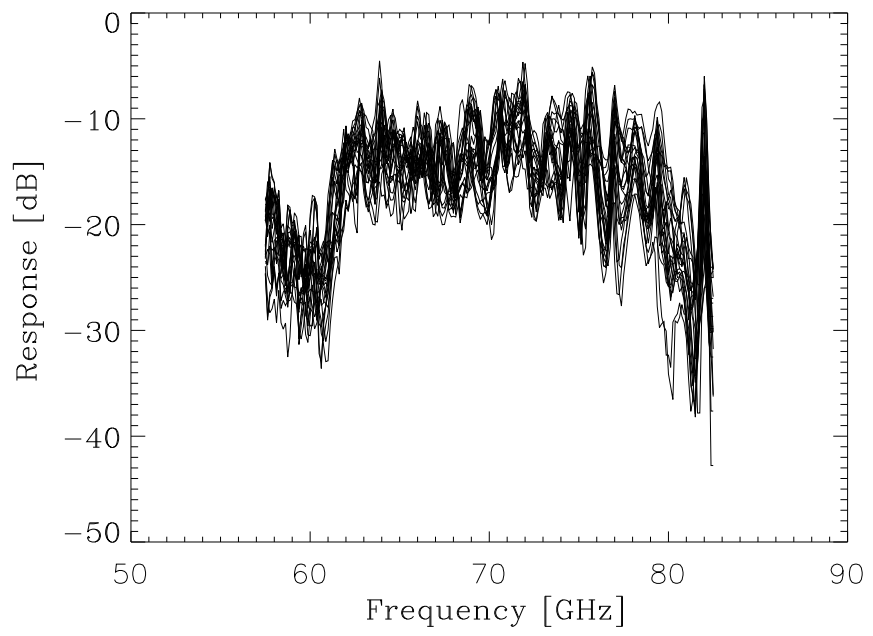

Fig. 18. Measured relative gain function (bandpass) of all 24 detectors at $77 \mathrm{GHz}$. The bandpasses are normalized to the area as explained in the text.

Table 7. Receiver noise properties. Long duration test antenna temperature pairs for the RCA28.

\begin{tabular}{ccccc}
\hline \hline \multirow{2}{*}{$T_{\text {sky }}[\mathrm{K}]$} & \multicolumn{2}{c}{$T_{\text {ref }}[\mathrm{K}]$} & \multicolumn{2}{c}{$\left(T_{\text {ref }}-T_{\text {sky }}\right)$} \\
& $\mathrm{M}$ & $\mathrm{S}$ & $\mathrm{M}$ & $\mathrm{S}$ \\
\hline 8.48 & 10.21 & 8.85 & 1.73 & 0.37 \\
9.07 & 15.16 & 14.66 & 6.10 & 5.59 \\
9.74 & 19.45 & 19.36 & 9.70 & 9.62 \\
19.63 & 19.45 & 19.36 & -0.18 & -0.27 \\
\hline
\end{tabular}

Table 8. Spectral response test results.

\begin{tabular}{llrrrr}
\hline \hline \multicolumn{7}{c}{ SPR BANDWIDTH AND CENTRAL FREQUENCY } \\
\multicolumn{1}{c}{} & M-00 & M-01 & S-10 & S-11 \\
\hline RCA18 & $\beta_{\text {spr }}$ & 12.40 & 11.14 & 10.84 & 10.25 \\
RCA19 & $\beta_{\text {spr }}$ & 10.45 & 10.74 & 8.00 & 9.91 \\
RCA20 & $\beta_{\text {spr }}$ & 11.19 & 12.21 & 12.57 & 10.82 \\
RCA21 & $\beta_{\text {spr }}$ & 11.19 & 12.21 & 12.57 & 10.82 \\
RCA22 & $\beta_{\text {spr }}$ & 11.49 & 10.38 & 11.11 & 10.44 \\
RCA23 & $\beta_{\text {spr }}$ & 10.35 & 11.52 & 11.62 & 11.44 \\
RCA24 & $\beta_{\text {spr }}$ & 5.15 & 4.08 & 5.26 & 5.82 \\
& $v_{0}$ & 45.75 & 42.4 & 45.6 & 45.3 \\
RCA25 & $\beta_{\text {spr }}$ & 4.42 & 4.49 & 4.17 & 5.91 \\
& $v_{0}$ & 45.75 & 45.25 & 45.85 & 44.90 \\
RCA26 & $\beta_{\text {spr }}$ & 6.10 & 4.86 & 4.26 & 5.48 \\
& $v_{0}$ & 44.35 & 44.85 & 44.90 & 44.20 \\
RCA27 & $\beta_{\text {spr }}$ & - & - & 3.89 & 3.71 \\
& $v_{0}$ & - & - & 30.45 & 30.70 \\
RCA28 & $\beta_{\text {spr }}$ & 4.94 & 5.12 & - & - \\
& $v_{0}$ & 31.4 & 31.35 & - & - \\
\hline
\end{tabular}

Notes. The numbers are bandwidth values, $\beta_{\text {spr }}$ and central frequency, $v_{0}$, both in $\mathrm{GHz}$.

There $G_{\text {tot }}\left(T_{0}^{\text {bem }}\right)$ is the total power gain when the BEM is at nominal physical temperature, $T_{0}^{\text {bem }}$, while $T^{\text {bem }}$ is the BEM physical temperature that was varied in steps. Non-linear effects were not considered because they were mainly caused by the change in $T_{\text {in }}$ which was fixed in this case. By exploiting the linear dependence between the voltage output and the BEM physical temperature as

$V_{\text {out }}=m \cdot T^{\text {bem }}+q$, 
Table 9. The transfer function of the susceptibility of FEM to temperature variations.

\begin{tabular}{lrrrr}
\hline \hline & \multicolumn{4}{c}{ FEM SUSCEPTIBILITY } \\
& M-00 & M-01 & \multicolumn{1}{c}{ S-10 } & \multicolumn{1}{c}{ S-11 } \\
\hline RCA18 & -8.48 & -9.13 & -6.77 & -7.67 \\
RCA19 & -9.43 & -9.28 & -1.20 & -9.49 \\
RCA20 & -6.61 & -5.69 & -5.93 & -5.79 \\
RCA21 & -3.01 & -1.85 & -0.770 & -0.930 \\
RCA22 & 5.67 & 5.21 & 5.69 & 6.04 \\
RCA23 & -2.07 & -4.41 & -4.07 & -3.92 \\
RCA24 & -1.21 & -0.610 & -2.03 & -0.964 \\
RCA25 & -1.51 & -1.33 & -2.87 & -2.21 \\
RCA26 & -6.22 & -6.10 & -6.57 & -6.31 \\
RCA27 & -1.68 & -1.05 & -3.64 & -3.06 \\
RCA28 & -0.266 & -0.519 & -1.85 & -1.05 \\
\hline
\end{tabular}

Table 10. The transfer function of the susceptibility of BEM to temperature variations.

\begin{tabular}{lrrrrr}
\hline \hline \multicolumn{7}{c}{ BEM SUSCEPTIBILITY } \\
TRANSFER FUNCTION 1/K \\
\hline M-00 & M-01 & S-10 & S-11 & $T_{0}^{\text {bem }, ~}{ }^{\circ} \mathrm{C}$ \\
RCA25 & -0.008 & -0.009 & -0.008 & -0.008 & 34.292 \\
RCA26 & -0.020 & -0.021 & -0.022 & -0.021 & 35.237 \\
RCA27 & -0.006 & -0.018 & -0.018 & -0.017 & 31.810 \\
\hline
\end{tabular}

Notes. The physical temperature of the BEM is also reported in the last column.

with the transfer function, $T_{\mathrm{f}}$, calculated from a linear fit to the data as

$T_{\mathrm{f}}=\frac{m}{m \cdot T_{0}^{\mathrm{bem}}+q}$.

The values are reported in Table 10.

\section{Conclusions}

The eleven LFI RCAs were calibrated according to the overall LFI calibration plan. The front end low-noise amplifiers and phase switches were properly tuned to guarantee minimum noise temperature and best isolation. Basic performances (noise temperature, isolation, linearity, photometric gain), noise properties ( $1 / f$ spectrum, noise equivalent bandwidth), relative bandshape, and susceptibility to thermal variations were measured in a dedicated cryogenic environment as close as possible to flight-operational conditions. All radiometric parameters were measured with excellent repeatability and reliability, except for $1 / f$ noise at $70 \mathrm{GHz}$ and some of the bandpasses at $30 \mathrm{GHz}$. The measurements were essentially in line with the design expectations, indicating a satisfactory instrument performance. Ultimately all RCA units were accepted, because the measured performances were in line with the scientific goal of LFI. The RCA test campaign described here represents the primary calibration test for some key radiometric parameters of the LFI, because they were not accurately measured as part of the RAA calibration campaign, nor can they be measured in-flight during the Planck nominal survey:

- Tuning results were used to set the subsequent tuning verification procedure up to the calibration performance and verification phase (CPV) in flight.
- Non-linear behavior of the 30 and $44 \mathrm{GHz}$ RCAs was accurately measured and characterized and used to estimate the impact in flight (Mennella et al. 2009). Moreover each radiometer system noise temperature was accurately determined.

- Except for some of the measured spectral responses, in which systematic effects arising from standing waves in the sky load were experienced, the RCA band shapes were only measured and characterized during the RCA tests. Together with the independent estimates of the band shape based on a synthesis of measured responses at unit level (Zonca et al. 2009), they are essential for the flight data analysis.

- Susceptivity to thermal variation of the FEM and BEM was measured and represents the reference values because only the FEM susceptivity was measured during RAA tests (Terenzi et al. 2009a), but under worse thermal conditions.

In conclusion we can state that even if the RCA calibration campaign was an intermediate step in the LFI development, the results obtained and presented here will be used in conjunction with the performance measured in flight to the exploitation of the scientific goal of Planck.

Acknowledgements. Planck is a project of the European Space Agency with instruments funded by ESA member states, and with special contributions from Denmark and NASA (USA). The Planck-LFI project is developed by an International Consortium lead by Italy and involving Canada, Finland, Germany, Norway, Spain, Switzerland, UK, USA. The Italian contribution to Planck is supported by the Italian Space Agency (ASI). In Finland, the Planck LFI $70 \mathrm{GHz}$ work was supported by the Finnish Funding Agency for Technology and Innovation (Tekes) T.P.'s work was supported in part by the Academy of Finland grants 205800, 214598, 121703, and 121962. T.P. thank Waldemar von Frenckells stiftelse, Magnus Ehrnrooth Foundation, and Väisälä Foundation for financial support. The Spanish participation is funded by Ministerio de Ciencia e Innovacion through the projects ESP2004-07067-C03-01 and AYA2007-68058C03-02.

\section{Appendix A: $Y$-factor method}

The classical $Y$-factor method is the simplest way to calculate the noise temperature, and it requires that radiometric data are acquired at two different (possibly well-separated) physical temperatures of one of the input loads. Below we will assume a sky load temperature increase. Clearly the treatment is completely symmetrical if the reference load temperature is increased. If we denote with $T_{\text {low }}$ and $T_{\text {high }}$ as the antenna temperatures corresponding to the skyload physical temperatures, we find that the ratio between the output voltages is given by

$\frac{V_{\text {low }}}{V_{\text {high }}}=\frac{T_{\text {low }}+T_{\mathrm{N}}}{T_{\text {high }}+T_{\mathrm{N}}} \equiv \frac{1}{Y}$

The system noise temperature is then calculated as

$T_{\mathrm{N}} \equiv \frac{T_{\mathrm{high}}-Y \cdot T_{\mathrm{low}}}{Y-1}$

\section{Appendix B: Radiometer non-linear model (gain model)}

A non-linear gain model was developed and applied to the 30 and $44 \mathrm{GHz}$ RCAs to model the observed behavior of the output voltages as a function of input temperature. The model was developed on the basis of Daywitt (1989) and specifically adopted for the LFI 30 and $44 \mathrm{GHz}$ RCAs, which exhibit a non-negligible 
compression effect in the BEMs. The FEM is assumed to have a constant gain and noise temperature

FEM: $\left\{\begin{array}{l}\text { Gain }=G_{0}^{\mathrm{FEM}} \\ \text { Noise }=T_{\mathrm{N}}^{\mathrm{FEM}} .\end{array}\right.$

The BEM (Artal et al. 2009) shows an overall gain (including the detector diode), which depends on the BEM input power as follows:

$\mathrm{BEM}:\left\{\begin{array}{l}\text { Gain }=G^{\mathrm{BEM}}=\frac{G_{0}^{\mathrm{BEM}}}{1+b \cdot G_{0}^{\mathrm{BEM}} \cdot p} \\ \text { Noise }=T_{\mathrm{N}}^{\mathrm{BEM}},\end{array}\right.$

where $p$ is the power entering the BEM and $b$ is a parameter defining the non-linearity of the BEM. For $b=0$ the radiometer is linear; for $b=\infty$ the BEM has a null-gain for any input power; for $p=\infty$ the BEM is completely compressed and $G^{\mathrm{BEM}}=0$ for any value of the non-linearity parameter.

The power entering the BEM (we here neglect the attenuation of the waveguides whose effect can be modeled as a small reduction of the FEM gain and a small increase of the FEM noise temperature) can be written as

$p=k \cdot B \cdot G_{0}^{\mathrm{FEM}} \cdot\left(T_{\mathrm{A}}+T_{\mathrm{N}}\right)$,

where

$T_{\mathrm{N}}=T_{\mathrm{N}}^{\mathrm{FEM}}+\frac{T_{\mathrm{N}}^{\mathrm{BEM}}}{G_{0}^{\mathrm{FEM}}}$.

The voltage at each output BEM detector (the detector diode constant is included in the BEM gain) can be written as

$V_{\text {out }}=k \cdot B \cdot G_{0}^{\mathrm{FEM}} \cdot \frac{G_{0}^{\mathrm{BEM}} \cdot\left(T_{\mathrm{A}}+T_{\mathrm{N}}\right)}{1+b \cdot G_{0}^{\mathrm{BEM}} \cdot\left(T_{\mathrm{A}}+T_{\mathrm{N}}\right)}$
$=G_{0}\left[\frac{1}{1+b \cdot G_{0} \cdot\left(T_{\mathrm{A}}+T_{\mathrm{N}}\right)}\right] \cdot\left(T_{\mathrm{A}}+T_{\mathrm{N}}\right)$,

where

$G_{0}=G_{0}^{\mathrm{FEM}} \cdot G_{0}^{\mathrm{BEM}} \cdot k \cdot B$.

In a compact way Eq. (B.6) can be written as

$$
\begin{aligned}
& V_{\text {out }}=G_{\mathrm{tot}} \cdot\left(T_{\mathrm{A}}+T_{\mathrm{N}}\right), \\
& G_{\text {tot }}=G_{0}\left[\frac{1}{1+b \cdot G_{0} \cdot\left(T_{\mathrm{A}}+T_{\mathrm{N}}\right)}\right],
\end{aligned}
$$

where the $G_{\text {tot }}$ is the total gain of the radiometer, which depends on the input antenna temperature; $G_{0}$ is the radiometer linear gain and it coincides with the overall gain in case of perfect linearity $(b=0)$.

\section{References}

Artal, E., Aja, B., de la Fuente, M. L., et al. 2009, JINST, 4, T12003 Bersanelli, M., Mandolesi, N., Butler, R. C., et al. 2010, A\&A, 520, A4
Cuttaia, F. 2005, Ph.D. Thesis, University of Bologna

Cuttaia, F., Mennella, A., Stringhetti, L., et al. 2009, JINST, 4, T12013 D’Arcangelo, O., Figini, L., Simonetto, A., et al. 2009a, JINST, 4, T12007

D’Arcangelo, O., Simonetto, A., Figini, L., Pagana, E., Villa, F., Pecora, M., Battaglia, P., Bersanelli, M., Butler, R. C., Garavaglia, S., Guzzi, P., Mandolesi, N., \& Sozzi, C. 2009b, JINST, 4, T12005

Davis, R. J., Wilkinson, A., Davies, R. D., et al. 2009, JINST, 4, T12002 Daywitt, W. C. 1989, NIST Tech. Note, 1327

De Nardo, S. 2008, Master's Thesis, Univeristà degli Studi di Milano Hoyland, R. 2004, US patent 6, 803, 838 B2

Malaspina, M., Franceschi, E., Battaglia, P., et al. 2009, JINST, 4, T12017

Mennella, A., Bersanelli, M., Seiffert, M., et al. 2003, A\&A, 410, 1089 Mennella, A., Villa, F., Terenzi, L., et al. 2009, JINST, 4, T12011

Mennella, A., Bersanelli, M., Butler, R. C., et al. 2010, A\&A, 520, A5

Seiffert, M., Mennella, A., Burigana, C., et al. 2002, A\&A, 391, 1185 Terenzi, L., Cuttaia, F., De Rosa, A. L. V., et al. 2009a, JINST, submitted Terenzi, L., Lapolla, M., Laaninen, M., et al. 2009b, JINST, 4, T12015 Terenzi, L., Salmon, M. J., Colin, A., et al. 2009c, JINST, 4, T12012 Valenziano, L., Cuttaia, F., De Rosa, A., et al. 2009, JINST, 4, T12006 Varis, J., Hughes, N. J., Laaninen, M., et al. 2009, JINST, 4, T12001 Villa, F., D’ Arcangelo, O., Pecora, M., et al. 2009, JINST, 4, T12004 Zonca, A., Franceschet, C., Battaglia, P., et al. 2009, JINST, 4, T12010

1 INAF - Istituto di Astrofisica Spaziale e Fisica Cosmica, via P. Gobetti, 101, 40129 Bologna, Italy e-mail: villa@iasfbo.inaf.it

2 Department of Physics, University of California, Santa Barbara, 93106-9530, USA

3 University of Helsinki, Department of Physics, PO Box 64, 00014 Helsinki, Finland

4 Helsinki Institute of Physics, PO Box 64, 00014 Helsinki, Finland

5 Metsähovi Radio Observatory, Helsinki University of Technology, Metsähovintie 114, 02540 Kylmälä, Finland

6 Thales Alenia Space Italia S.p.A., S.S. Padana Superiore 290, 20090 Vimodrone (MI), Milano, Italy

7 Università degli Studi di Milano, via Celoria 16, 20133 Milano, Italy

8 DA-Design Oy. (aka Ylinen Electronics), Keskuskatu 29, 31600 Jokioinen, Finland

9 IFP-CNR, via Cozzi 53, 20125 Milano, Italy

10 INAF - Osservatorio Astronomico di Trieste, 11 via Tiepolo, 34143 Trieste, Italy

11 University of Trieste, Department of Physics, 2 via Valerio, 34127 Trieste, Italy

12 Jodrell Bank Centre for Astrophysics, Alan Turing Building, The University of Manchester, Manchester, M13 9PL, UK

13 Planck Science Office, European Space Agency, ESAC, PO box 78, 28691 Villanueva de la Cañada, Madrid, Spain

14 INAF - Istituto di Astrofisica Spaziale e Fisica Cosmica, via E. Bassini 15, 20133 Milano, Italy

15 Universidad de Cantabria, Dep. De Ingenieria de Comunicaciones. Av. Los Castros s/n, 39005, Santander-Spain

16 Instituto de Fisica de Cantabria (CSIC-UC), Av. Los Castros s/n, 39005 Santander, Spain

17 Jet Propulsion Laboratory, 4800 Oak Grove Drive, Pasadena, California 91109, USA

18 Instituto de Astrofísica de Canarias C/, Via Lactea s/n, 38205 La Laguna (Tenerife), Spain

19 National Radio Astronomy Observatory, Stone hall, University of Virginia, 520 Edgemont road, Charlottesville, USA

20 MilliLab, VTT Technical Research Centre of Finland, Tietotie 3, Otaniemi, Espoo, Finland 